\title{
Applications of the pseudo residual-free bubbles to the stabilization of the convection-diffusion-reaction problems in 2D
}

\author{
A. Sendur ${ }^{a}$, A. Nesliturk ${ }^{\text {b,* }}$, A. Kaya ${ }^{b}$ \\ ${ }^{a}$ Department of Mathematics Education, Akdeniz University, 07490 Antalya, Turkey \\ ${ }^{\mathrm{b}}$ Department of Mathematics, Izmir Institute of Technology, 35430 Izmir, Turkey
}

Received 11 December 2012; received in revised form 23 April 2014; accepted 26 April 2014

Available online 6 May 2014

\begin{abstract}
A stabilized finite element method is studied herein for two-dimensional convection-diffusion-reaction problems. The method is based on the residual-free bubbles (RFB) method. However we replace the RFB functions by their cheap, yet efficient approximations computed on a specially chosen subgrid, which retain the same qualitative behavior. Since the correct spot of subgrid points plays a crucial role in the approximation, it is important to determine their optimal locations, which we do it through a minimization process with respect to the $L_{1}$-norm. The resulting numerical method has similar stability features with the well-known stabilized methods in the literature for the whole range of problem parameters and this fact is also confirmed by numerical experiments.
\end{abstract}

(C) 2014 Elsevier B.V. All rights reserved.

Keywords: Stabilized finite element methods; Pseudo residual-free bubbles; Augmented space

\section{Introduction}

The convection-diffusion-reaction (CDR) equation and its particular cases provide very useful and important mathematical model in wide range of applications including natural sciences and engineering. Those problems may exhibit layer structures when the diffusion term is highly dominated by the convection or the reaction terms which is challenging from the numerical point of view. In these cases, it is well known that, the plain Galerkin method does not work on reasonable discretizations and unphysical oscillations appear throughout the domain. Therefore developing efficient and effective computational methods for solving the CDR problems has been drawing attention of many researchers for several decades.

More accurate and stable results can be obtained using the stabilized methods, which are based on augmenting the variational formulation by mesh-dependent terms in order to gain control over the derivatives of the numerical solution. The Streamline-Upwind Petrov-Galerkin (SUPG) method is the most popular one providing a consistent and stabilizing numerical scheme for convection-diffusion problems [1-3]. The Galerkin/least-squares (GLS) method represents a conceptual generalization of the SUPG method and its essential

\footnotetext{
* Corresponding author. Tel.: +90 2327507756.

E-mail addresses: alisendur@akdeniz.edu.tr (A. Sendur), alinesliturk@iyte.edu.tr, anesli@yahoo.com (A. Nesliturk), ademkaya@iyte.edu.tr (A. Kaya).
} 
feature is to augment the Galerkin finite element formulation with a least-squares form of the residuals that are based on the corresponding differential equations [4]. A review of various successful approaches for the convection-diffusion equation can be found in [5] and references therein. Variations of the SUPG method such as GLS and gradient Galerkin/least-squares (GGLS) methods have been extended to the convection-diffusion-reaction problems in [6-9]. Further attempts to develop accurate and stable results in the presence of reactive terms are introduced with the unusual stabilized finite element method (USFEM) by Franca et al. [10,11]. The 'unusual' feature of this stabilized method is the subtraction of a mesh dependent term from the standard Galerkin method. A thorough comparison of some of these methods can be found in [12]. The advantage of these approaches is not only their generality, but also their computer implementation is quite practical. Though their widespread popularity, the stabilized methods were criticized for the lack of theoretical background as they could not be derived from the fundamental principles. Furthermore, the stability parameters in these methods are obtained either from the error analysis or from the experiments by the trial and error as there is no general methodology to determine their optimal value.

In his significant works $[13,14]$, Hughes proposed the Variational Multiscale Method (VMS), with which the origins of the stabilized methods can be enlightened and the different classes of stabilized methods can be put together in a theoretical setting. The basic idea of the Variational Multiscale approach is to decompose the solution into coarse and fine scale that tries to determine the fine scale solution analytically or numerically and eliminate it from the problem for the coarse scale solution. The variational multiscale approach provides not only a theoretical background for the stabilized methods, but also guidelines and inspiration for the development and improvement of many different stabilization techniques and numerical methods [15-18].

One of the well-known stabilization techniques that can be related to the Variational Multiscale framework is the residual-free bubbles (RFB) method. This method is based on a local enrichment of the finite element space with special type of bubble functions, so called the residual-free bubbles [19-24], whose relation to the stabilized and the variational multiscale methods was studied in [25-27], respectively. Although the RFBs form an effective platform to derive improved discretizations, they are defined by a set of local differential equations posed inside each element which may not be easier to solve than the original one, except that the problem domains are simple element geometries (they are either triangle or quadrangle). Regarding that fact, it was proposed a more recent approach that relies on constructing a small-sized subgrid inside each element and approximate bubble functions on it. At this point, it is worth mentioning that a similar numerical algorithm in the context of the variational multiscale approach was successfully applied to the convection-diffusion problem in [17,18]. The good performance of the RFB methodology has been shown not only for the convection-diffusion-reaction problems in one space dimension [28], but also for the convection-diffusion problems in two space dimensions [29,30]. However, its extension to the convection-diffusion-reaction problems in two space dimensions is not straightforward.

A similar approach in the framework of the RFB method, yet applicable in higher dimensions was proposed for the convection-diffusion problems by Brezzi et al. in [31]. In this approach the RFB functions are again replaced by approximate bubbles consisting of piecewise linears, called pseudo residual-free bubbles, on a suitably chosen subgrid inside each element. Here the locations of subgrid points are of critical importance and therefore they should be chosen specially so that fine scale-effect of the exact solution can accurately be represented in the coarse scale numerical approximation. In particular, their location is determined by minimizing the residual of a local differential problem defining the bubbles, with respect to the $L_{1}$-norm. This approach has been successfully applied to twodimensional convection-diffusion problems in [32] and one-dimensional convection-diffusion-reaction problems in [33].

The merit of the paper is to extend the stabilization technique above to the convection-diffusion-reaction problems in two space dimensions, especially in the cases of small diffusion. The subgrid nodes are derived for the convectiondominated and the reaction-dominated cases separately, however they are able to adapt from one regime to another smoothly and continuously. Numerical experiments show good performance of the proposed method and that the resulting numerical method has good stability features. In turn, we can get the numerical solutions at almost the RFB quality, yet, in a less expensive manner. It is also worth pointing out that the internal nodes in convection-dominated case take the form of the optimal locations of the subgrid points suggested by Brezzi et al. in [29] in a similar framework showing that the proposed method is consistent with the previous results.

The layout of the paper is as follows. In Section 2, we briefly recall the basic ideas of the RFB method. We discuss how to construct a subgrid on which we describe the pseudo bubble functions in Section 3. In Section 4, we describe 
the details of the numerical method proposed and perform the numerical tests for the convection-dominated cases. The corresponding results for the reaction-dominated cases are discussed in Section 5.

\section{Stabilization through augmented spaces}

We will consider the following linear elliptic convection-diffusion-reaction problem in a polygonal domain $\Omega$ :

$$
\left\{\begin{array}{l}
\mathcal{L} u=-\epsilon \Delta u+\beta \cdot \nabla u+\sigma u=f \quad \text { in } \Omega \\
u=0 \quad \text { on } \partial \Omega .
\end{array}\right.
$$

Let $\mathcal{T}_{h}=\{K\}$ be a decomposition of $\Omega$ into triangles $K$, and let $h_{K}=\operatorname{diam}(K)$ with $h=\max _{K \in \mathcal{T}_{h}} h_{K}$. We assume that the diffusion coefficient $\epsilon$ is a positive constant, the convection field $\beta$ and the reaction field $\sigma$ are non-negative piecewise constants with respect to the decomposition $\mathcal{T}_{h}$. Here we will consider a stabilization method based on the augmented space idea in the context of the RFB strategy. To describe that approach, we first recall the variational formulation of the problem (1): Find $u \in H_{0}^{1}(\Omega)$ such that

$$
a(u, v)=(f, v) \quad \forall v \in H_{0}^{1}(\Omega)
$$

where

$$
a(u, v)=\epsilon \int_{\Omega} \nabla u \cdot \nabla v+\int_{\Omega}(\beta \cdot \nabla u) v+\int_{\Omega} \sigma u v .
$$

Define a finite-dimensional subspace $V_{h}$ of $H_{0}^{1}(\Omega)$. Then the standard Galerkin finite element method reads: Find $u_{h} \in V_{h}$ such that

$$
a\left(u_{h}, v_{h}\right)=\left(f, v_{h}\right) \quad \forall v_{h} \in V_{h} .
$$

Now, we decompose the space $V_{h}$ such that $V_{h}=V_{L} \bigoplus V_{B}$, where $V_{L}$ is the space of continuous piecewise linear polynomials and $V_{B}=\bigoplus_{K} B_{K}$ with $B_{K}=H_{0}^{1}(K)$. We require the bubble component $u_{B}$ of $u_{h}$ to satisfy the original differential equation in $K$ strongly, i.e.

$$
\mathcal{L} u_{B}=-\mathcal{L} u_{L}+f \quad \text { in } K \quad \text { subject to the boundary condition } \quad u_{B}=0 \quad \text { on } \partial K .
$$

By means of the classical static condensation procedure [28], the method used to compute an improved linear approximation due to the residual-free bubble effect reads: Find $u_{h}=u_{L}+u_{B}$ in $V_{h}$ such that

$$
a\left(u_{L}, v_{L}\right)+a\left(u_{B}, v_{L}\right)=\left(f, v_{L}\right) \quad \forall v_{L} \in V_{L} .
$$

The term $a\left(u_{B}, v_{L}\right)$ is responsible for the stabilization of the numerical method and the bubble component $u_{B}$ should be computed before we solve (6) for its linear part. However, recall that $u_{B}$ is identified by the linear part $u_{L}$ and the source function $f$ through (5), whose solutions may be as complicated as solving the original differential equation. Therefore, it is important to have a simple algorithm to obtain a suitable approximation to $u_{B}$, that provides a similar stabilizing effect into the numerical method. Regarding the simplicity of element geometry, this approach can be turned into a workable method, whose details are given in the following section.

\section{The pseudo residual-free bubbles}

Let $P_{1}, P_{2}$ and $P_{3}$ be internal points of $K$, for which we will construct pseudo bubble functions. The quality of approximate bubble functions, which is crucial to get a good stabilization effect on the numerical method, is essentially related with the location of those internal points. Therefore the choice of those points must be fulfilled in a special manner. That will be accomplished through a minimization process with respect to the $L_{1}$-norm in the presence of layers.

To be more descriptive, we consider bubble functions $B_{i},(i=1,2,3)$ defined by

$$
\mathcal{L} B_{i}=-\mathcal{L} \psi_{i} \quad \text { in } K, \quad B_{i}=0 \quad \text { on } \partial K, \quad i=1,2,3
$$




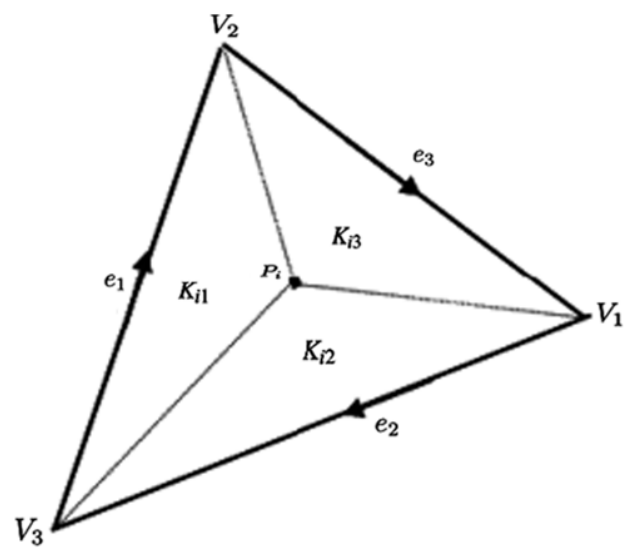

Fig. 1. Splitting $K$ into three sub-regions by $P_{i}$.

where $\psi_{i}$ are the restrictions of the piecewise linear basis functions for $V_{L}$ to $K$. Further we define $B_{f}$,

$$
\mathcal{L} B_{f}=f \quad \text { in } K, \quad B_{f}=0 \quad \text { on } \partial K .
$$

Now if

$$
\left.u_{L}\right|_{K}=\sum_{i=1}^{3} c_{i} \psi_{i} \quad \text { then }\left.u_{B}\right|_{K}=\sum_{i=1}^{3} c_{i} B_{i}+B_{f}
$$

with the same coefficient $c_{i}$. Thus

$$
\mathcal{L} u_{B}=-\mathcal{L} u_{L}+f \quad \text { in } K .
$$

That is, Eq. (5) is automatically satisfied with the present choice of bubble functions. Eq. (7) is similar to the original problem (1) and its solution may be a difficult task. However, using the element geometry and the problem properties, it is possible to construct less expensive, yet efficient approximate bubbles, say $B_{i}^{*}$, having the same qualitative behavior with its continuous counterparts. The construction of such approximate bubble functions $B_{i}^{*}$ is introduced in the following.

Let $b_{i}$ be a piecewise linear function with

$$
b_{i}\left(V_{j}\right)=0 \quad \text { and } \quad b_{i}\left(P_{i}\right)=1 \quad \forall i, j=1,2,3
$$

where $V_{i}$ are the vertices of $K$. Further let $B_{i}^{*}(x)=\alpha_{i} b_{i}(x)$ be the classical Galerkin approximation of $B_{i}$ through (7)

$$
a\left(B_{i}^{*}, b_{i}\right)_{K}=\left(-\mathcal{L} \psi_{i}, b_{i}\right)_{K}, \quad i=1,2,3 .
$$

An easy computation from (9) gives

$$
\alpha_{i}=\frac{\left(-\mathcal{L} \psi_{i}, b_{i}\right)}{\epsilon\left\|\nabla b_{i}\right\|_{K}^{2}+\sigma\left\|b_{i}\right\|_{K}^{2}}, \quad i=1,2,3 .
$$

The main criteria that we use to determine the locations of the internal points is to minimize the $L_{1}$-norm of the residual coming out from the bubble Eq. (7) in the critical case where a layer structure exists. In other words, we choose $P_{i}$ such that

$$
J_{i}=\int_{K}\left|\mathcal{L} B_{i}^{*}+\mathcal{L} \psi_{i}\right|, \quad i=1,2,3
$$

is minimum. Before we derive the explicit locations of the internal points, additional notation related to the element geometry should be introduced first. We denote the edges of $K$ by $e_{i}$ opposite to $V_{i}$, the length of $e_{i}$ by $\left|e_{i}\right|$, the midpoint of edge $e_{i}$ by $M_{i}$, the outward unit normal to $e_{i}$ by $n^{i}, v_{i}=\left|e_{i}\right| n^{i}$ and $\beta_{v_{i}}=\left(\beta, v_{i}\right)$ (see Fig. 1). We 

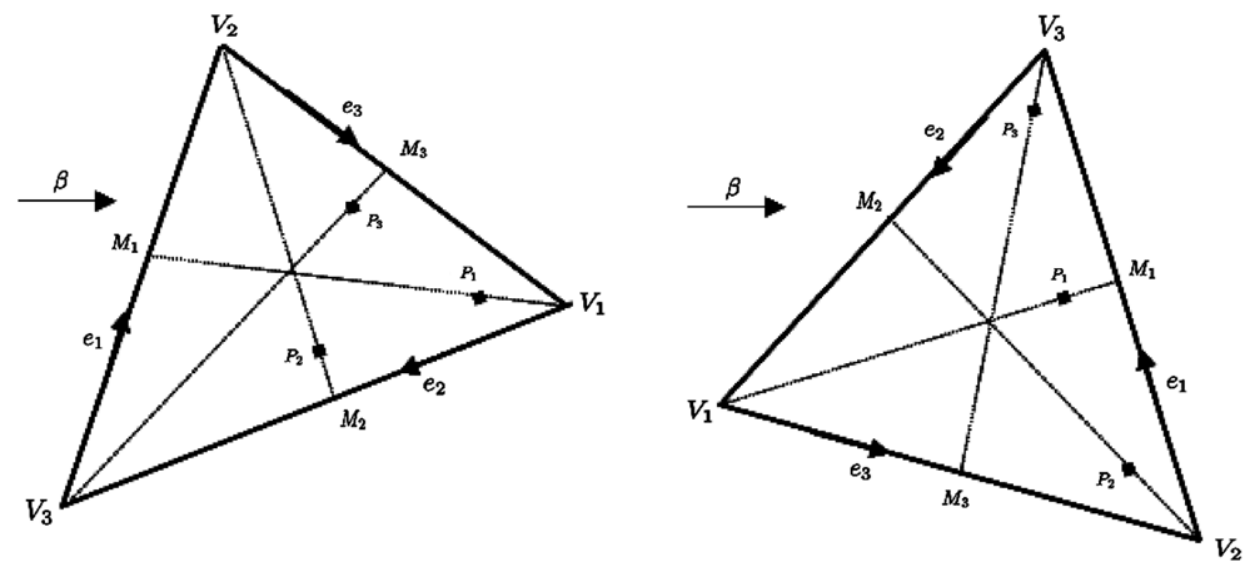

Fig. 2. Configuration of internal nodes in convection-dominated regime: one inflow edge (left) and two inflow edges (right).

further introduce the three sub-triangles $K_{i 1}, K_{i 2}, K_{i 3}$ which are obtained by connecting the additional node $P_{i}$ with the vertices of $K$ and the area of $K_{i j}$ by $\left|K_{i j}\right|$. The actual numbering of the vertices will be chosen according to the direction of $\beta$.

Now, let us choose the location of $P_{i}$ along the median from $V_{i}$, that is

$$
P_{i}=\left(1-t_{i}\right) M_{i}+t_{i} V_{i}, \quad 0<t_{i}<1, \quad i=1,2,3 .
$$

In order to specify the problem regimes, we further introduce

$$
\epsilon_{i}^{*}=\frac{2|K|\left(-3 \beta_{v_{i}}+\sigma|K|\right)}{9\left(\left|e_{1}\right|^{2}+\left|e_{2}\right|^{2}+\left|e_{3}\right|^{2}\right)} \quad i=1,2,3 .
$$

Now we are in a position to determine the explicit locations of subgrid points in each problem regime.

\section{Convection-dominated flows}

To be able to determine the explicit locations of the internal points, we have to distinguish among the following cases: (see Fig. 2).

\subsection{One inflow edge}

Let the inflow boundary make up of one edge and let $e_{1}$ be the inflow one. Then, we have $\beta_{\nu_{1}}<0, \beta_{\nu_{2}}>0$ and $\beta_{\nu_{3}}>0$. We assume that the problem is convection-dominated if

$$
\epsilon \leq \epsilon_{1}^{*} \quad \text { with } 2 \sigma|K|<\min \left\{\beta_{\nu_{2}}, \beta_{\nu_{3}}\right\} .
$$

Now, split $K$ into three subregions by using the definition of $P_{1}$ in (12) (see Fig. 1), so that we have

$$
\left|K_{11}\right|=t_{1}|K| \quad\left|K_{12}\right|=\left|K_{13}\right|=\left(1-t_{1}\right)|K| / 2 .
$$

Moreover, we have

$$
\psi_{1}=\left.\frac{\left|K_{11}\right|}{|K|} b_{1}\right|_{K_{11}} \quad \text { and }\left.\quad \nabla b_{1}\right|_{K_{1 i}}=-\left|e_{i}\right| n_{i} / 2\left|K_{1 i}\right|, \quad i=1,2,3 .
$$


Further, define

$$
l_{i j k}(t)=\left|e_{i}\right|^{2}+t\left|e_{j}-e_{k}\right|^{2} \quad \text { and } \quad f_{i j k}(t)=3 \epsilon l_{i j k}(t)+2(1-t) t \sigma|K|^{2}, \quad i, j, k=1,2,3 .
$$

Note that $l_{i j k}(t) \sim O\left(h^{2}\right)$ for any $t \in(0,1)$. Now, using the properties of bubble function $b_{1}$ and the midpoint rule for quadratic terms that appears, we get an explicit expression for $\alpha_{1}$ depending on the parameter $t_{1}$ :

$$
\alpha_{1}=\frac{\left(-\mathcal{L} \psi_{1}, b_{1}\right)}{\epsilon\left\|\nabla b_{1}\right\|_{K}^{2}+\sigma\left\|b_{1}\right\|_{K}^{2}}=-\frac{\left(S_{1}+t_{1} \sigma|K|\right) / 12}{\frac{\epsilon l_{123}\left(t_{1}\right)}{4\left(1-t_{1}\right) t_{1}|K|}+\frac{\sigma|K|}{6}}=-\frac{t_{1}\left(1-t_{1}\right)|K|\left(S_{1}+t_{1} \sigma|K|\right)}{f_{123}\left(t_{1}\right)}
$$

where $S_{1}=-2 \beta_{\nu_{1}}+\sigma|K|$. Note that $S_{1}>0$ and $\alpha_{1}<0$. Before finding an optimal position for $P_{1}$, we need the following intermediate result.

Lemma 1. $\int_{K}\left|-\epsilon \Delta B_{1}^{*}\right|$ is an increasing function of $t_{1}$ in the interval $(1 / 2,1)$.

Proof. Following the lines of [31], we have

$$
\int_{K}\left|-\epsilon \Delta B_{1}^{*}\right|=-\epsilon \alpha_{1} \sum_{i=1}^{3} \frac{\left|e_{i}\right|^{2}}{2\left|K_{1 i}\right|} .
$$

Define

$$
\tilde{f}_{i j k}(t)=2|K|\left(S_{i}\left((2 t-1)\left|e_{i}\right|^{2}+t^{2}\left|e_{j}-e_{k}\right|^{2}\right)+2 t^{2} \sigma|K|\left(\left|e_{j}\right|^{2}+\left|e_{k}\right|^{2}\right)\right) .
$$

Now, use the expression for $\alpha_{1}$ in (14) to get

$$
\begin{aligned}
\frac{d}{d t_{1}} \int_{K}\left|-\epsilon \Delta B_{1}^{*}\right| & =-\frac{d}{d t_{1}}\left(\epsilon \alpha_{1}\left(\frac{\left|e_{1}\right|^{2}}{2\left|K_{11}\right|}+\frac{\left|e_{2}\right|^{2}}{2\left|K_{12}\right|}+\frac{\left|e_{3}\right|^{2}}{2\left|K_{13}\right|}\right)\right) \\
& =\epsilon \frac{d}{d t_{1}}\left(\frac{t_{1}\left(1-t_{1}\right)\left(S_{1}+t_{1} \sigma|K|\right)}{f_{123}\left(t_{1}\right)}\left(\frac{\left|e_{1}\right|^{2}}{2 t_{1}}+\frac{\left|e_{2}\right|^{2}}{1-t_{1}}+\frac{\left|e_{3}\right|^{2}}{1-t_{1}}\right)\right) \\
& =\epsilon \frac{d}{d t_{1}}\left(\frac{S_{1}+t_{1} \sigma|K|}{f_{123}\left(t_{1}\right)}\left(\frac{1-t_{1}}{2}\left|e_{1}\right|^{2}+t_{1}\left|e_{2}\right|^{2}+t_{1}\left|e_{3}\right|^{2}\right)\right) \\
& =\epsilon \sigma|K| \frac{3 \epsilon l_{123}^{2}\left(t_{1}\right)+\tilde{f}_{123}\left(t_{1}\right)}{2 f_{123}^{2}\left(t_{1}\right)} .
\end{aligned}
$$

Hence the result immediately follows since $f_{123}\left(t_{1}\right)>0$ and $\tilde{f}_{123}\left(t_{1}\right)>0$ whenever $1 / 2<t_{1}<1$.

The following lemma suggests an optimal position for $P_{1}$ along the median from $V_{1}$ by using (11).

Theorem 2. If the inflow boundary make up of one edge, then the point

$$
t_{1}^{*}=1-\frac{-\rho_{1}+\sqrt{\rho_{1}^{2}+\lambda_{1}}}{2 \sigma|K|^{2}}
$$

minimizes the integral $J_{1}$ in convection-dominated flows where

$$
\rho_{1}=-2 \beta_{\nu_{1}}|K|+3 \epsilon\left|e_{2}-e_{3}\right|^{2}, \quad \lambda_{1}=24 \epsilon \sigma|K|^{2}\left(\left|e_{2}\right|^{2}+\left|e_{3}\right|^{2}\right) .
$$

Proof. It is possible to rewrite the integral $J_{1}$ in (11) as follows (see [31]):

$$
J_{1}=\int_{K}\left|-\epsilon \Delta B_{1}^{*}\right|+\sum_{i=1}^{3} \int_{K_{1 i}}\left|\beta \cdot \nabla B_{1}^{*}+\sigma B_{1}^{*}+\beta \cdot \nabla \psi_{1}+\sigma \psi_{1}\right| .
$$


Let $g_{1}=\beta \cdot \nabla B_{1}^{*}+\sigma B_{1}^{*}+\beta \cdot \nabla \psi_{1}+\sigma \psi_{1}$. When an appropriate form of $\alpha_{1}$ in (14) is substituted, we get

$$
\begin{aligned}
\int_{K} g_{1} & =-\frac{1}{2} \beta_{v_{1}}+\frac{\sigma|K|}{3}\left(\alpha_{1}+1\right) \\
& =-\frac{1}{2} \beta_{v_{1}}+\frac{\sigma|K|}{3}\left(-\frac{t_{1}\left(1-t_{1}\right)|K|\left(S_{1}+t_{1} \sigma|K|\right)}{f_{123}\left(t_{1}\right)}+1\right) \\
& =\frac{3 \epsilon l_{123}\left(t_{1}\right)\left(-3 \beta_{v_{1}}+2 \sigma|K|\right)+2\left(1-t_{1}\right) t_{1} \sigma|K|^{2}\left(-\beta_{v_{1}}+\left(1-t_{1}\right) \sigma|K|\right)}{6 f_{123}\left(t_{1}\right)}
\end{aligned}
$$

where the last expression is obviously positive. Regarding the splitting of $K$ by $P_{1}$ (Fig. 1), we investigate the sign of $g_{1}$ over each of these sub-domains to get:

$$
\begin{aligned}
\left.g_{1}\right|_{K_{12}} & =\left.\left(\beta \cdot \nabla B_{1}^{*}+\sigma B_{1}^{*}+\beta \cdot \nabla \psi_{1}+\sigma \psi_{1}\right)\right|_{K_{12}} \\
& =\left.\alpha_{1} \beta \cdot \nabla b_{1}\right|_{K_{12}}+\left.\alpha_{1} \sigma b_{1}\right|_{K_{12}}+\left.\frac{\left|K_{11}\right|}{|K|} \beta \cdot \nabla b_{1}\right|_{K_{11}}+\sigma \psi_{1} \\
& =-\frac{\alpha_{1}}{2\left|K_{12}\right|} \beta_{\nu_{2}}+\left.\alpha_{1} \sigma b_{1}\right|_{K_{12}}-\frac{\beta_{\nu_{1}}}{2|K|}+\sigma \psi_{1} \\
& >-\frac{\alpha_{1}}{2\left|K_{12}\right|} \beta_{\nu_{2}}+\alpha_{1} \sigma-\frac{\beta_{\nu_{1}}}{2|K|}+\sigma \psi_{1} \quad\left(\text { since } \max _{\mathbf{x} \in K_{12}} b_{1}(\mathbf{x})<1\right) \\
& >-\frac{\alpha_{1}}{2\left|K_{12}\right|} \beta_{\nu_{2}}+\alpha_{1} \sigma \quad\left(\text { since } \max _{\mathbf{x} \in K_{12}} \psi_{1}(\mathbf{x})>0 \text { and } \beta_{\nu_{1}}<0\right) \\
& =-\alpha_{1}\left(\frac{\beta_{\nu_{2}}}{2\left|K_{12}\right|}-\sigma\right)
\end{aligned}
$$

which is positive by condition (13). Similarly, we have

$$
\left.g_{1}\right|_{K_{13}}=\left.\left(\beta \cdot \nabla B_{1}^{*}+\sigma B_{1}^{*}+\beta \cdot \nabla \psi_{1}+\sigma \psi_{1}\right)\right|_{K_{13}}>-\alpha_{1}\left(\frac{\beta_{\nu_{3}}}{2\left|K_{13}\right|}-\sigma\right)>0 .
$$

Thus, the second term on the right hand side of (15) attains its minimum if $\left.g_{1}\right|_{K_{11}}$ is positive, too:

$$
\begin{aligned}
\left.g_{1}\right|_{K_{11}} & =\left.\left(\beta \cdot \nabla B_{1}^{*}+\sigma B_{1}^{*}+\beta \cdot \nabla \psi_{1}+\sigma \psi_{1}\right)\right|_{K_{11}} \\
& =\left.\alpha_{1} \beta \cdot \nabla b_{1}\right|_{K_{11}}+\left.\alpha_{1} \sigma b_{1}\right|_{K_{11}}+\left.\frac{\left|K_{11}\right|}{|K|} \beta \cdot \nabla b_{1}\right|_{K_{11}}+\sigma \psi_{1} \\
& =-\frac{\alpha_{1}}{2\left|K_{11}\right|} \beta_{\nu_{1}}+\left.\alpha_{1} \sigma b_{1}\right|_{K_{11}}-\frac{\beta_{\nu_{1}}}{2|K|}+\sigma \psi_{1} \\
& =\left(\alpha_{1}+t_{1}\right)\left(-\frac{\beta_{\nu_{1}}}{2|K| t_{1}}+\left.\sigma b_{1}\right|_{K_{11}}\right) \\
& =-\frac{t_{1} \sigma|K|^{2}\left(t_{1}-1+\frac{-\rho_{1}+\sqrt{\rho_{1}^{2}+\lambda_{1}}}{2 \sigma|K|^{2}}\right)\left(t_{1}-1-\frac{\rho_{1}+\sqrt{\rho_{1}^{2}+\lambda_{1}}}{2 \sigma|K|^{2}}\right)}{3 \epsilon l_{123}\left(t_{1}\right)+2 t_{1}\left(1-t_{1}\right) \sigma|K|^{2}}\left(-\frac{\beta_{\nu_{1}}}{2|K| t_{1}}+\left.\sigma b_{1}\right|_{K_{11}}\right) .
\end{aligned}
$$

The only root of the last expression in $(0,1)$ is $t_{1}^{*}=1-\frac{-\rho_{1}+\sqrt{\rho_{1}^{2}+\lambda_{1}}}{2 \sigma|K|^{2}}$ and $\left.g_{1}\right|_{K_{11}}$ is positive if $t_{1} \geq t_{1}^{*}$. This fact together with Lemma 1 determines an optimal value for $t_{1}$. 
Remark 1. As the actual value of $t_{1}$, we do not always take that given by Theorem 2. Indeed, for $\epsilon$ not too small (that is, for diffusion dominated problems) this type of stabilization would be unnecessary. Furthermore the value provided by Theorem 2 can even be meaningless since it is derived in the presence of layers. Therefore, we take

$$
t_{1}= \begin{cases}t_{1}^{*}, & \text { if } \epsilon \leq \epsilon_{1}^{*} \\ 1 / 3, & \text { otherwise. }\end{cases}
$$

Notice that for $\epsilon=\epsilon_{1}^{*}$ we have exactly $t_{1}=t_{1}^{*}=1 / 3$ and for $0<\epsilon<\epsilon_{1}^{*}$ we have $1>t_{1}^{*}>1 / 3$. Therefore for every $\epsilon>0$, we have $\frac{1}{3} \leq t_{1}<1$. This can be seen as a weaker assumption than we made in Lemma 1 , that was $t_{1} \in(1 / 2,1)$. However, it seems just as a technicality as we get reasonable approximations for $t_{1}$ in $(1 / 3,1)$.

Remark 2. In the limiting case of pure convection, we observe that

$$
\lim _{\sigma \rightarrow 0} t_{1}^{*}=1-\frac{6 \epsilon\left(\left|e_{2}\right|^{2}+\left|e_{3}\right|^{2}\right)}{-2 \beta_{\nu_{1}}|K|+3 \epsilon\left|e_{2}-e_{3}\right|^{2}},
$$

that is, $t_{1}^{*}$ takes the form of the optimal point suggested by Brezzi et al. in [29] for the convection-diffusion problems.

Remark 3. The value of $\alpha_{1}$ at $t_{1}^{*}$ is simply equal to $-t_{1}^{*}$.

Remark 4. For a convection dominated regime, the choice of other points, $P_{2}$ and $P_{3}$, should be consistent with the physics of the problem. Thus we take

$$
t_{1}=t_{1}^{*}, \quad t_{2}=t_{3}=\min \left\{1 / 3,1-t_{1}^{*}\right\} .
$$

\subsection{Two inflow edges}

Let the inflow boundary make up of two edges and let $e_{2}$ and $e_{3}$ be the inflow ones. Then, we have $\beta_{\nu_{1}}>0, \beta_{\nu_{2}}<0$ and $\beta_{v_{3}}<0$. In this case, we assume that the problem is convection-dominated if

$$
\epsilon \leq \min \left\{\epsilon_{2}^{*}, \epsilon_{3}^{*}\right\} \quad \text { with } 2 \sigma|K|<\beta_{\nu_{1}} .
$$

Now, we will construct the explicit locations of $P_{2}$ and $P_{3}$, separately:

\subsubsection{Construction of $P_{2}$ and $P_{3}$}

Similarly, we determine the locations of $P_{2}$ and $P_{3}$ along the medians from $V_{2}$ and $V_{3}$, respectively. Splitting $K$ by $P_{2}$ and $P_{3}$, we have

$$
\begin{array}{lll}
\left|K_{21}\right|=\left|K_{23}\right|=\left(1-t_{2}\right)|K| / 2 & \text { and } & \left|K_{22}\right|=t_{2}|K|, \\
\left|K_{31}\right|=\left|K_{32}\right|=\left(1-t_{3}\right)|K| / 2 & \text { and } & \left|K_{33}\right|=t_{3}|K| .
\end{array}
$$

The explicit expressions for $\alpha_{2}$ and $\alpha_{3}$ :

$$
\begin{aligned}
& \alpha_{2}=\frac{\left(-\mathcal{L} \psi_{2}, b_{2}\right)}{\epsilon\left\|\nabla b_{2}\right\|_{K}^{2}+\sigma\left\|b_{2}\right\|_{K}^{2}}=-\frac{\left(S_{2}+t_{2} \sigma|K|\right) / 12}{\frac{\epsilon l_{213}\left(t_{2}\right)}{4\left(1-t_{2}\right) t_{2}|K|}+\frac{\sigma|K|}{6}}=-\frac{t_{2}\left(1-t_{2}\right)|K|\left(S_{2}+t_{2} \sigma|K|\right)}{f_{213}\left(t_{2}\right)} \\
& \alpha_{3}=\frac{\left(-\mathcal{L} \psi_{3}, b_{3}\right)}{\epsilon\left\|\nabla b_{3}\right\|_{K}^{2}+\sigma\left\|b_{3}\right\|_{K}^{2}}=-\frac{\left(S_{3}+t_{3} \sigma|K|\right) / 12}{\frac{\epsilon l_{312}\left(t_{3}\right)}{4\left(1-t_{3}\right) t_{3}|K|}+\frac{\sigma|K|}{6}}=-\frac{t_{3}\left(1-t_{3}\right)|K|\left(S_{3}+t_{3} \sigma|K|\right)}{f_{312}\left(t_{3}\right)}
\end{aligned}
$$


where $S_{2}=-2 \beta_{\nu_{2}}+\sigma|K|$ and $S_{3}=-2 \beta_{\nu_{3}}+\sigma|K|$. Note that $S_{2}>0, \alpha_{2}<0$ and $S_{3}>0, \alpha_{3}<0$. Before finding the optimal positions for $P_{2}$ and $P_{3}$, one need the following:

Lemma 3. $\int_{K}\left|-\epsilon \Delta B_{2}^{*}\right|$ and $\int_{K}\left|-\epsilon \Delta B_{3}^{*}\right|$ are increasing functions of $t_{2}$ and $t_{3}$, respectively, in the interval $(1 / 2,1)$.

Proof. The proof is similar to Lemma 1, so we skip it.

The following lemma suggests an optimal position for $P_{2}$ and $P_{3}$ by using (11).

Theorem 4. If the inflow boundary make up of two edges then the points $t_{2}^{*}=1-\frac{-\rho_{2}+\sqrt{\rho_{2}^{2}+\lambda_{2}}}{2 \sigma|K|^{2}}$ and $t_{3}^{*}=$ $1-\frac{-\rho_{3}+\sqrt{\rho_{3}^{2}+\lambda_{3}}}{2 \sigma|K|^{2}}$ minimize the integrals $J_{2}$ and $J_{3}$, respectively, in convection-dominated flows where

$$
\begin{aligned}
& \rho_{2}=-2 \beta_{\nu_{2}}|K|+3 \epsilon\left|e_{1}-e_{3}\right|^{2}, \quad \lambda_{2}=24 \epsilon \sigma|K|^{2}\left(\left|e_{1}\right|^{2}+\left|e_{3}\right|^{2}\right) \\
& \rho_{3}=-2 \beta_{\nu_{3}}|K|+3 \epsilon\left|e_{1}-e_{2}\right|^{2}, \quad \lambda_{3}=24 \epsilon \sigma|K|^{2}\left(\left|e_{1}\right|^{2}+\left|e_{2}\right|^{2}\right) .
\end{aligned}
$$

Proof. The proof is similar to the one of Theorem 2. Recall that

$$
J_{2}=\int_{K}\left|-\epsilon \Delta B_{2}^{*}\right|+\sum_{i=1}^{3} \int_{K_{2 i}}\left|\beta \cdot \nabla B_{2}^{*}+\sigma B_{2}^{*}+\beta \cdot \nabla \psi_{2}+\sigma \psi_{2}\right| .
$$

Let $g_{2}=\beta \cdot \nabla B_{2}^{*}+\sigma B_{2}^{*}+\beta \cdot \nabla \psi_{2}+\sigma \psi_{2}$. A direct calculation over $K$ gives,

$$
\begin{aligned}
\int_{K} g_{2} & =-\frac{1}{2} \beta_{\nu_{2}}+\frac{\sigma|K|}{3}\left(\alpha_{2}+1\right) \\
& =\frac{3 \epsilon l_{213}\left(t_{2}\right)\left(-3 \beta_{\nu_{2}}+2 \sigma|K|\right)+2\left(1-t_{2}\right) t_{2} \sigma|K|^{2}\left(-\beta_{\nu_{2}}+\left(1-t_{2}\right) \sigma|K|\right)}{6 f_{213}\left(t_{2}\right)}
\end{aligned}
$$

where the last expression is obviously positive. Now split $K$ into three subregions by $P_{2}$ and investigate the sign of $g_{2}$ over each of these sub-domains:

$$
\begin{aligned}
\left.g_{2}\right|_{K_{21}} & =\left.\left(\beta \cdot \nabla B_{2}^{*}+\sigma B_{2}^{*}+\beta \cdot \nabla \psi_{2}+\sigma \psi_{2}\right)\right|_{K_{21}} \\
& =\left.\alpha_{2} \beta \cdot \nabla b_{2}\right|_{K_{21}}+\left.\alpha_{2} \sigma b_{2}\right|_{K_{21}}+\left.\frac{\left|K_{22}\right|}{|K|} \beta \cdot \nabla b_{2}\right|_{K_{22}}+\sigma \psi_{2} \\
& =-\frac{\alpha_{2}}{2\left|K_{21}\right|} \beta_{\nu_{1}}+\left.\alpha_{2} \sigma b_{2}\right|_{K_{21}}-\frac{\beta_{\nu_{2}}}{2|K|}+\sigma \psi_{2} \\
& >-\frac{\alpha_{2}}{2\left|K_{21}\right|} \beta_{\nu_{1}}+\alpha_{2} \sigma-\frac{\beta_{\nu_{2}}}{2|K|}+\sigma \psi_{2} \quad\left(\text { since } \max _{\mathbf{x} \in K_{21}} b_{2}(\mathbf{x})<1\right) \\
& >-\frac{\alpha_{2}}{2\left|K_{21}\right|} \beta_{v_{1}}+\alpha_{2} \sigma \quad\left(\text { since } \max _{\mathbf{x} \in K_{21}} \psi_{2}(\mathbf{x})>0 \text { and } \beta_{\nu_{2}}<0\right) \\
& =-\alpha_{2}\left(\frac{\beta_{v_{1}}}{2\left|K_{21}\right|}-\sigma\right)
\end{aligned}
$$

which is positive by condition (17). Similarly, we have 


$$
\begin{aligned}
\left.g_{2}\right|_{K_{23}} & =\left.\left(\beta \cdot \nabla B_{2}^{*}+\sigma B_{2}^{*}+\beta \cdot \nabla \psi_{2}+\sigma \psi_{2}\right)\right|_{K_{23}} \\
& =\left.\alpha_{2} \beta \cdot \nabla b_{2}\right|_{K_{23}}+\left.\alpha_{2} \sigma b_{2}\right|_{K_{23}}+\left.\frac{\left|K_{22}\right|}{|K|} \beta \cdot \nabla b_{2}\right|_{K_{22}}+\sigma \psi_{2} \\
& =-\frac{\alpha_{2}}{2\left|K_{23}\right|} \beta_{\nu_{3}}+\left.\alpha_{2} \sigma b_{2}\right|_{K_{23}}-\frac{\beta_{\nu_{2}}}{2|K|}+\left.\sigma \frac{\left|K_{22}\right|}{|K|} b_{2}\right|_{K_{22}} \\
& <-\frac{\alpha_{2}}{2\left|K_{23}\right|} \beta_{\nu_{3}}-\frac{\beta_{\nu_{2}}}{2|K|}+\sigma \frac{\left|K_{22}\right|}{|K|}\left(\text { since } 0<\max _{\mathbf{x} \in K_{22} \cup K_{23}} b_{2}(\mathbf{x})<1\right) \\
& =\frac{l_{213}\left(t_{2}\right)\left(\epsilon-\tilde{\epsilon}_{2}\left(t_{2}\right)\right)\left(-\beta_{\nu_{2}}+2 t_{2} \sigma|K|\right)}{2|K| f_{213}\left(t_{2}\right)}
\end{aligned}
$$

where $\tilde{\epsilon}_{2}(t)=\frac{2|K| t\left(-\beta_{\nu_{3}}\left(-2 \beta_{\nu_{2}}+(1+t) \sigma|K|\right)-(1-t) \sigma|K|\left(-\beta_{\nu_{2}}+2 t \sigma|K|\right)\right)}{3 l_{213}(t)\left(-\beta_{\nu_{2}}+2 t \sigma|K|\right)}$. We note that the sign of the previous expression only depends on the sign of $\epsilon-\tilde{\epsilon}_{2}\left(t_{2}\right)$. According to the observation below

$$
\begin{aligned}
\tilde{\epsilon}_{2}(t) & =\frac{2|K| t\left(-\beta_{\nu_{3}}\left(-2 \beta_{\nu_{2}}+(1+t) \sigma|K|\right)-(1-t) \sigma|K|\left(-\beta_{\nu_{2}}+2 t \sigma|K|\right)\right)}{3 l_{213}(t)\left(-\beta_{\nu_{2}}+2 t \sigma|K|\right)} \\
> & \frac{|K|\left(-\beta_{\nu_{3}}\left(-2 \beta_{\nu_{2}}+\frac{3}{2} \sigma|K|\right)-\sigma|K|\left(-\frac{\beta_{\nu_{2}}}{2}+\sigma|K|\right)\right)}{6\left(\left|e_{1}\right|^{2}+\left|e_{3}\right|^{2}\right)\left(-\beta_{\nu_{2}}+2 \sigma|K|\right)} \quad(\text { since } 1 / 2<t<1) \\
> & \frac{|K|\left(-2 \beta_{\nu_{2}}+\frac{3}{2} \sigma|K|\right)\left(-\beta_{\nu_{3}}-\frac{2}{3} \sigma|K|\right)}{6\left(\left|e_{1}\right|^{2}+\left|e_{3}\right|^{2}\right)\left(-\beta_{\nu_{2}}+2 \sigma|K|\right)} \quad\left(\text { since }-\frac{\beta_{\nu_{2}}}{2}+\sigma|K|<\frac{2}{3}\left(-2 \beta_{\nu_{2}}+\frac{3}{2} \sigma|K|\right)\right) \\
> & \frac{|K|\left(-\beta_{\nu_{3}}-\frac{2}{3} \sigma|K|\right)}{8\left(\left|e_{1}\right|^{2}+\left|e_{3}\right|^{2}\right)} \quad\left(\text { since }-2 \beta_{\nu_{2}}+\frac{3}{2} \sigma|K|>\frac{3}{4}\left(-\beta_{\nu_{2}}+2 \sigma|K|\right)\right),
\end{aligned}
$$

$\epsilon-\tilde{\epsilon}_{2}\left(t_{2}\right)$ will apparently be negative in convection-dominated flows, which implies that $\left.g_{2}\right|_{K_{23}}<0$. Thus, the second term on the right hand side of (20) attains its minimum if $\left.g_{2}\right|_{K_{22}}$ is non-negative, ${ }^{1}$ too:

$$
\begin{aligned}
\left.g_{2}\right|_{K_{22}} & =\left.\left(\beta \cdot \nabla B_{2}^{*}+\sigma B_{2}^{*}+\beta \cdot \nabla \psi_{2}+\sigma \psi_{2}\right)\right|_{K_{22}} \\
& =\left.\alpha_{2} \beta \cdot \nabla b_{2}\right|_{K_{22}}+\left.\alpha_{2} \sigma b_{2}\right|_{K_{22}}+\left.\frac{\left|K_{22}\right|}{|K|} \beta \cdot \nabla b_{2}\right|_{K_{22}}+\sigma \psi_{2} \\
& =\left(\alpha_{2}+t_{2}\right)\left(-\frac{\beta_{\nu_{2}}}{2|K| t_{2}}+\left.\sigma b_{2}\right|_{K_{22}}\right) \\
& =-\frac{t_{2} \sigma|K|^{2}\left(t_{2}-1+\frac{-\rho_{2}+\sqrt{\rho_{2}^{2}+\lambda_{2}}}{2 \sigma|K|^{2}}\right)\left(t_{2}-1-\frac{\rho_{2}+\sqrt{\rho_{2}^{2}+\lambda_{2}}}{2 \sigma|K|^{2}}\right)}{3 \epsilon l_{213}\left(t_{2}\right)+2 t_{2}\left(1-t_{2}\right) \sigma|K|^{2}}\left(-\frac{\beta_{\nu_{2}}}{2|K| t_{2}}+\left.\sigma b_{2}\right|_{K_{22}}\right) .
\end{aligned}
$$

The only root of the last expression in $(0,1)$ is $t_{2}^{*}=1-\frac{-\rho_{2}+\sqrt{\rho_{2}^{2}+\lambda_{2}}}{2 \sigma|K|^{2}}$ and $\left.g_{2}\right|_{K_{22}}$ is positive if $t_{2} \geq t_{2}^{*}$. This fact together with Lemma 3 determines an optimal value for $t_{2}$.

The proof for $t_{3}$ is similarly obtained by just changing the roles of $e_{2}$ and $e_{3}$, and replacing subindex 2 by subindex 3 , accordingly.

\footnotetext{
${ }^{1}$ Let $a, b, c \in R, a+b+c>0, a>0$ and $c<0$ are given. Try to minimize $|a|+|b|+|c|$ : since $c<0$ we have $a+b-c>a+b+c>0$ and therefore $a-c>-b$ and $|a|+|b|+|c|=a+|b|-c>-b+|b|= \begin{cases}-2 b, & \text { if } b<0 \\ 0, & \text { if } b>0\end{cases}$
} 

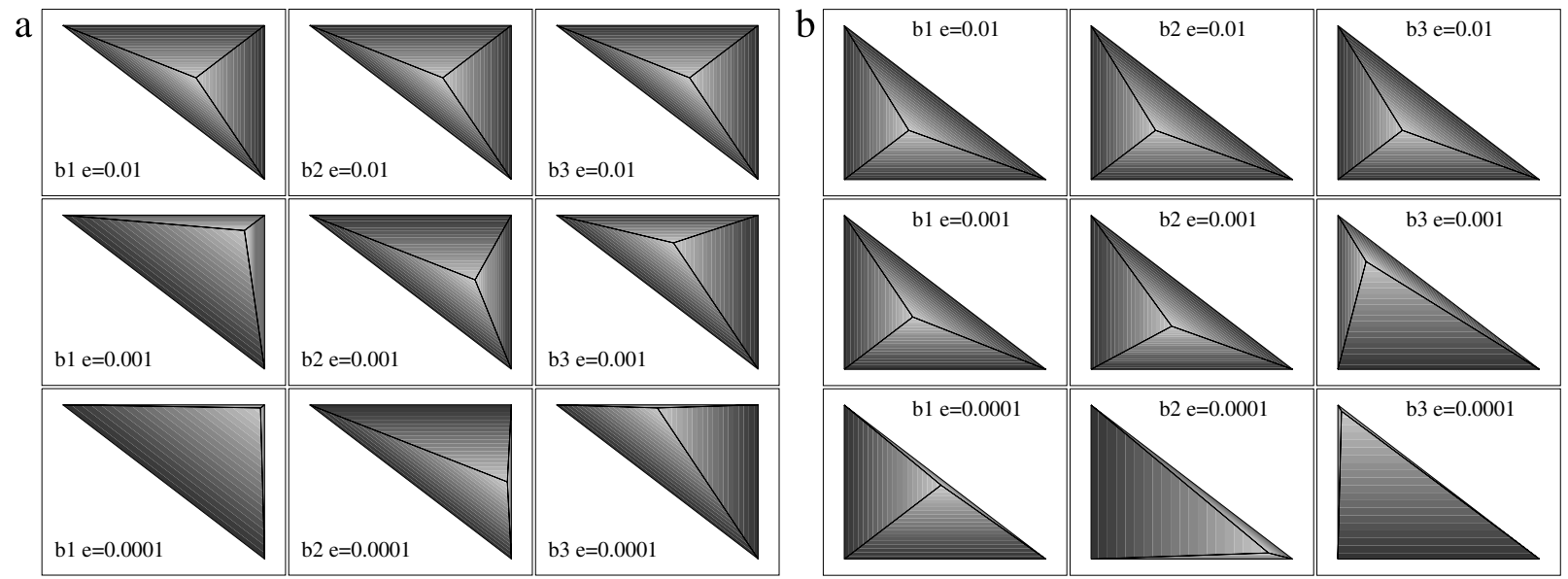

Fig. 3. Bubble functions, $b_{1}, b_{2}, b_{3}$, in a typical element having (a) one inflow edge (left), (b) two inflow edges (right), when $\theta=72^{\circ}, N=20$, $\epsilon=10^{-2}, 10^{-3}, 10^{-4}$.

Remark 5. The stabilization is unnecessary for diffusion dominated problems. Therefore, we take

$$
t_{2}=\left\{\begin{array}{ll}
t_{2}^{*}, & \text { if } \epsilon \leq \epsilon_{2}^{*}, \\
1 / 3, & \text { otherwise }
\end{array} \text { and } t_{3}= \begin{cases}t_{3}^{*}, & \text { if } \epsilon \leq \epsilon_{3}^{*}, \\
1 / 3, & \text { otherwise }\end{cases}\right.
$$

which also give a continuous dependence of $t_{2}$ and $t_{3}$ upon $\epsilon$. Also note that, we have $\frac{1}{3} \leq t_{2}, t_{3}<1, \forall \epsilon>0$.

Remark 6. Observe that the points $t_{2}^{*}$ and $t_{3}^{*}$ turn into the optimal points suggested by Brezzi et al. in [29] for the convection-diffusion problems, that is,

$$
\lim _{\sigma \rightarrow 0} t_{2}^{*}=1-\frac{6 \epsilon\left(\left|e_{1}\right|^{2}+\left|e_{3}\right|^{2}\right)}{-2 \beta_{v_{2}}|K|+3 \epsilon\left|e_{1}-e_{3}\right|^{2}} \quad \text { and } \quad \lim _{\sigma \rightarrow 0} t_{3}^{*}=1-\frac{6 \epsilon\left(\left|e_{1}\right|^{2}+\left|e_{2}\right|^{2}\right)}{-2 \beta_{v_{3}}|K|+3 \epsilon\left|e_{2}-e_{1}\right|^{2}} .
$$

Remark 7. The values of $\alpha_{2}$ at $t_{2}^{*}$ and $\alpha_{3}$ at $t_{3}^{*}$ are simply equal to $-t_{2}^{*}$ and $-t_{3}^{*}$, respectively.

Remark 8. In the present configuration, the choice of $P_{1}$ should be consistent with the physics of the problem. Thus we take

$$
t_{2}=t_{2}^{*}, \quad t_{3}=t_{3}^{*}, \quad t_{1}=\min \left\{1 / 3,1-t_{2}^{*}, 1-t_{3}^{*}\right\} .
$$

Remark 9. In Fig. 3, we display the behavior of approximate bubble functions in two types of element configuration with one inflow edge and with two inflow edges, for various intensities of diffusion $\left(\epsilon=10^{-2}, 10^{-3}, 10^{-4}\right)$ when $\theta=72^{\circ}$ and $\sigma=0.001$. The first columns of the figures present the bubble function, $b_{1}$, for decreasing values of diffusion $\left(\epsilon=10^{-2}, 10^{-3}, 10^{-4}\right)$. The corresponding numerical results for $b_{2}$ and $b_{3}$ are shown in column 2 and 3 , respectively. Note the self-adjustment of the subgrid nodes as the problem evolves from the diffusion-dominated regime into the convection-dominated one. The locations of subgrid nodes are automatically adjusted between the problem regimes so that the pseudo bubbles contribute to the stability of the numerical method at their most.

\subsection{Numerical experiments}

In this section, we report some numerical experiments to illustrate the performance of the present algorithm in convection-dominated flows. We also report the $L_{2}$ and $H_{1}$ errors. 


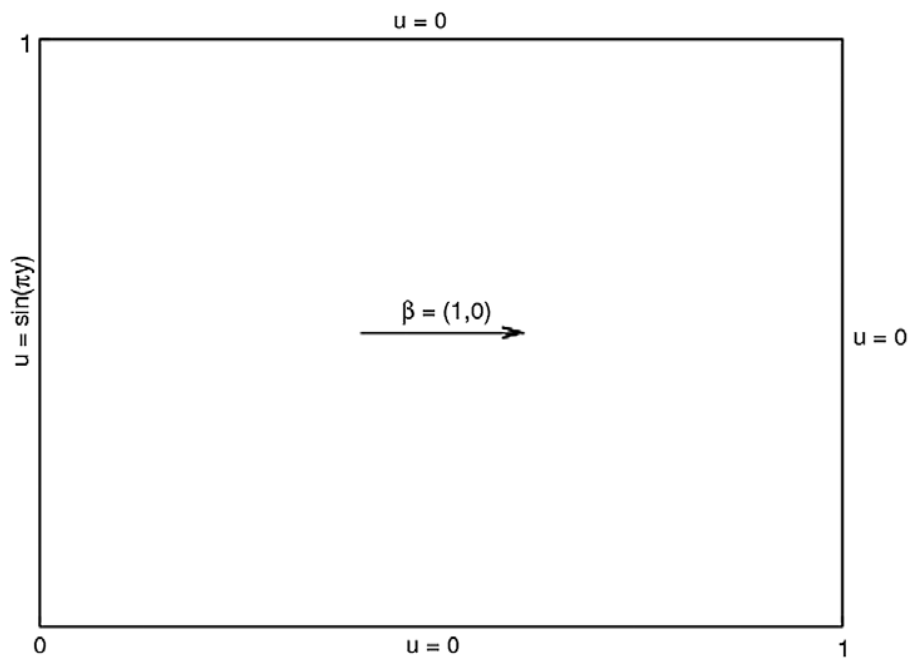

Fig. 4. Configuration of Test problem 4.3.1.
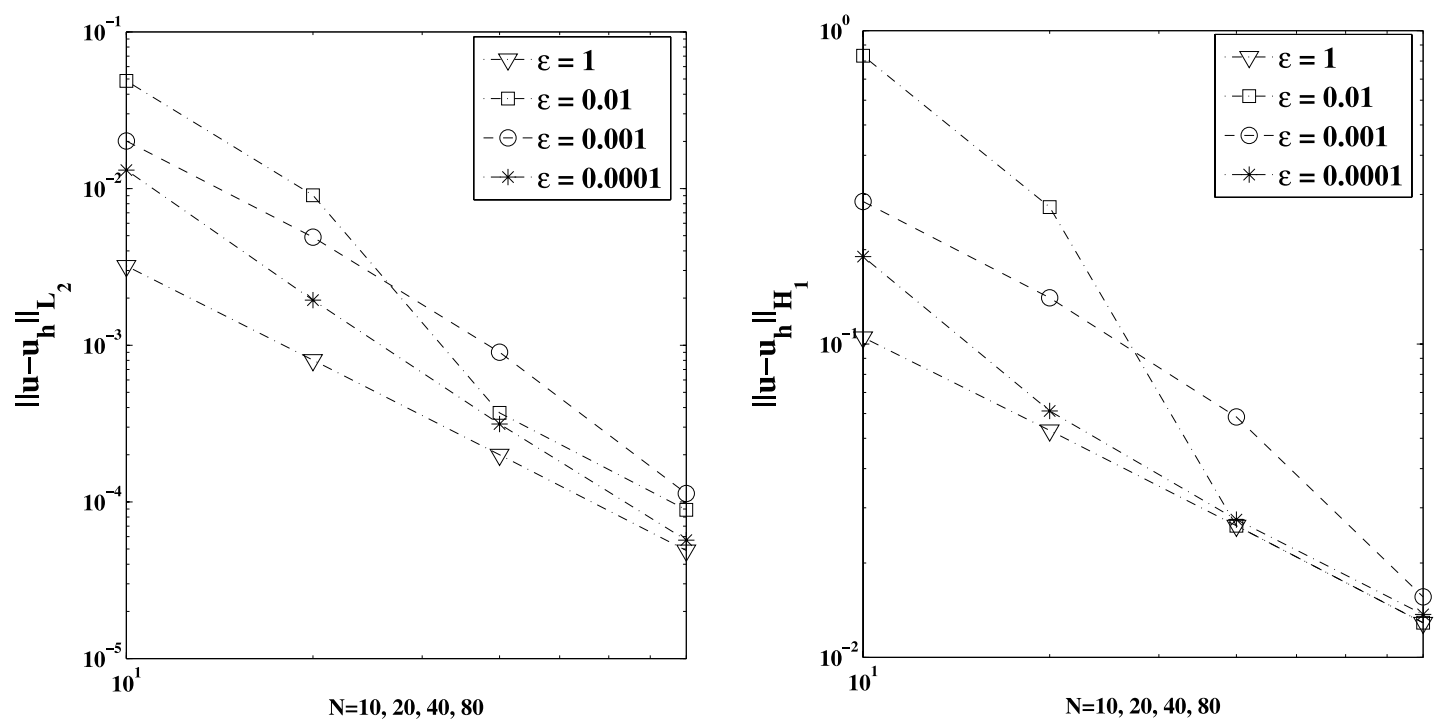

Fig. 5. The error in $L_{2}$ (left) and $H_{1}$ (right) norms for several values of $\epsilon$ when $\beta=(1,0), \sigma=0.001$ and $f(x)=0$.

\subsubsection{An example with analytical solution}

In the convergence analysis, we consider a simple problem on a unit square that can be solved analytically [21]. Consider the unit square subject to the following boundary conditions (see Fig. 4 for problem statement):

$$
u=\left\{\begin{array}{l}
0, \quad \text { if } y=0, \quad 0 \leq x \leq 1, \\
0, \quad \text { if } x=1, \quad 0 \leq y \leq 1, \\
0, \quad \text { if } y=1, \quad 0 \leq x \leq 1, \\
\sin (\pi y), \quad \text { if } x=0, \quad 0 \leq y \leq 1 .
\end{array}\right.
$$

We set $\beta=(1,0)$ and $f(x)=0$ in $\Omega$. Using separation of variables, the exact solution is given by:

$$
u(x, y)=\frac{e^{x / 2 \epsilon} \sinh (-m(1-x)) \sin (\pi y)}{\sinh (-m)} \quad \text { where } m=\sqrt{1+4 \epsilon\left(\epsilon \pi^{2}+\sigma\right)} / 2 \epsilon .
$$

Next, we take a set of uniform triangular meshes which are made up of $N=10,20,40$ elements, respectively, in $x$ and $y$ directions. In Fig. 5, we present the log-log plots of errors in $L_{2}$ and $H_{1}$ norms for different values 


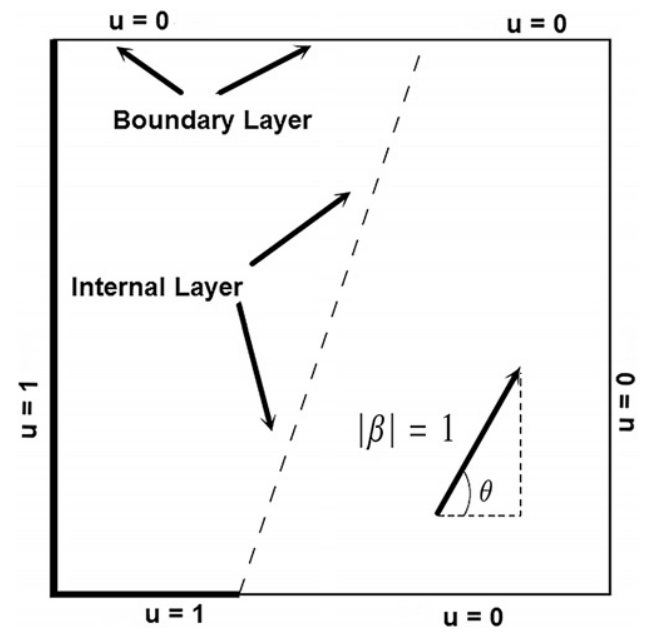

Fig. 6. Configuration of Test problem 4.3.2.

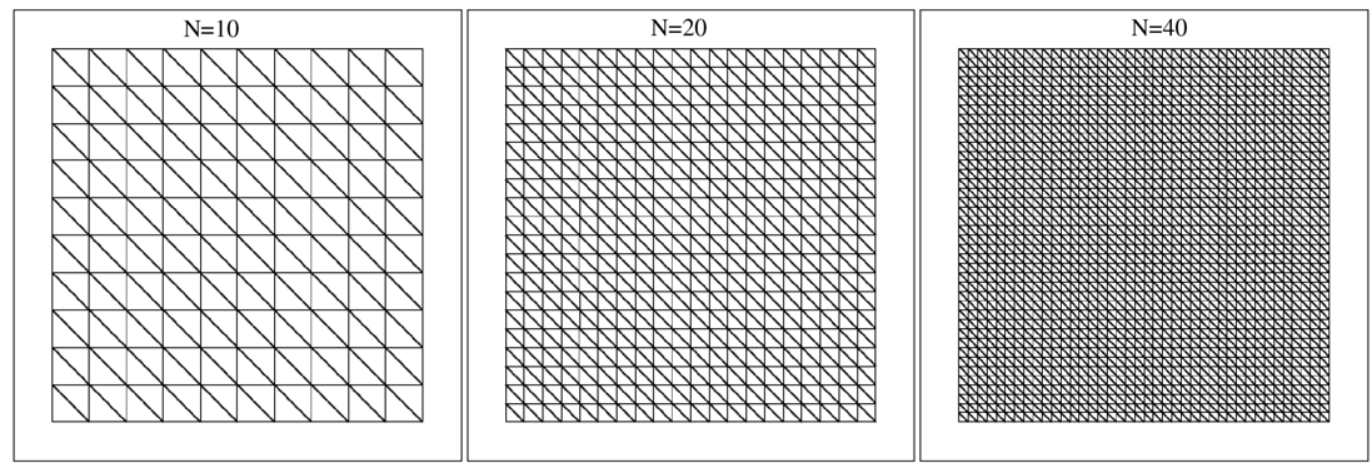

Fig. 7. Triangular elements used in discretization of the problem domain.

of the mesh parameter $\mathrm{N}$ when $\sigma=0.001$ and $\epsilon=1,10^{-2}, 10^{-3}, 10^{-4}$. Although the improvement is apparent as the mesh is refined, a slight degradation in the approximation can be observed for parameter values in the mid-regime.

\subsubsection{Propagation of discontinuity}

We consider a test case shown in Fig. 6, in which the exact solution exhibits an internal and a boundary layer [34].

We first take a set of uniform triangular meshes which are made up of $N=10,20,40$ elements, respectively, in $x$ and $y$ directions (Fig. 7). In Figs. 8-10, we plot the solutions obtained with the present method for several values of $\theta$ when the convection dominates the flow, that is, $\epsilon=0.001, \sigma=0.001$ and $f=0$. Although the present method could not eliminate the oscillations at all, it captures the layers well, even on coarse meshes.

Next, we consider the same problem on a set of non-uniform triangular meshes which are made up of $N=$ 10, 20, 40 elements, respectively, in $x$ and $y$ directions (see Fig. 11(a)). In Fig. 11(b), we display the numerical results obtained with the present method for $\theta=72^{\circ}, \epsilon=0.01, \sigma=0.001$ and $f=0$. The numerical solutions show that the method is robust as the results are consistent with the physical configuration of the problem. 

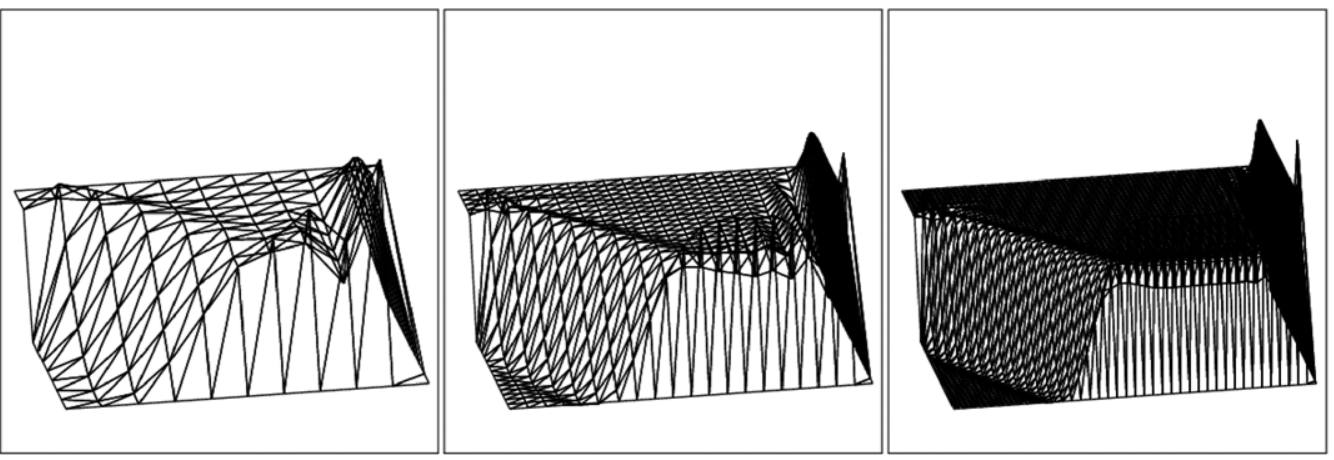

(a)
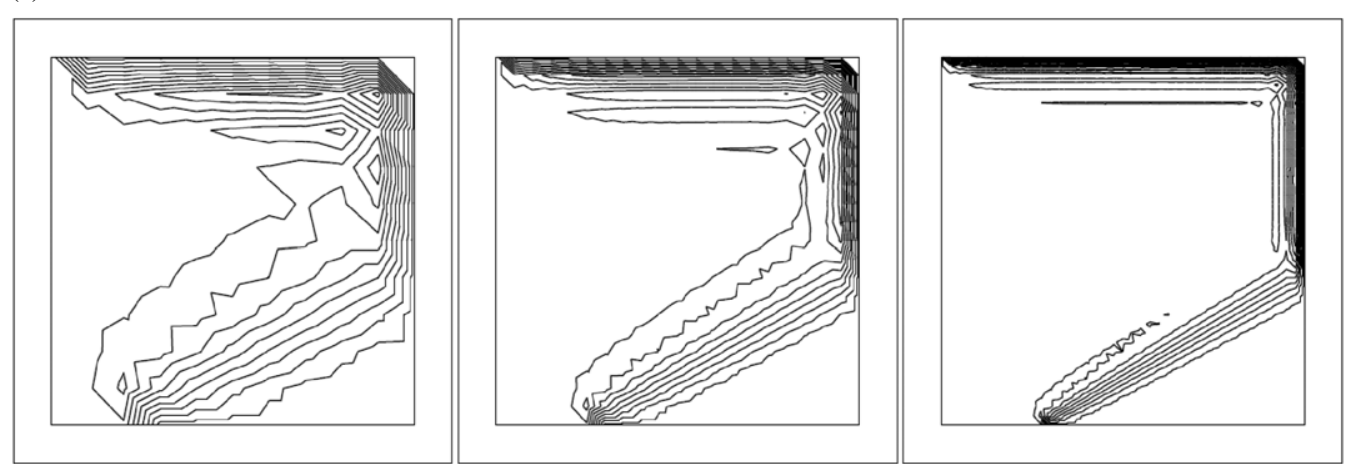

(b)

Fig. 8. Numerical solutions for $\theta=30$ (left to right $N=10,20,40$ ) and the corresponding contour plots.
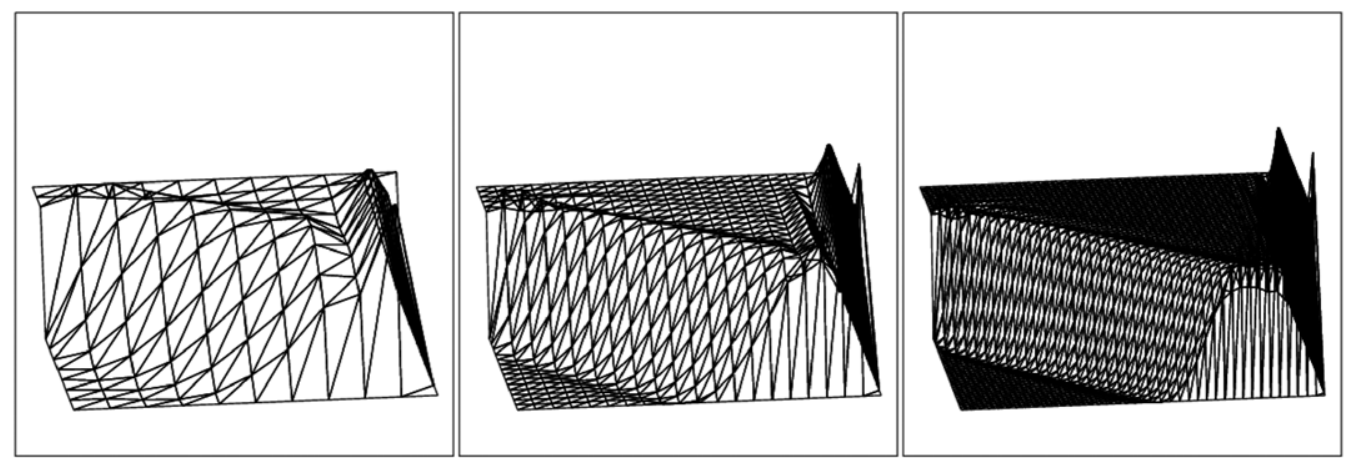

(a)
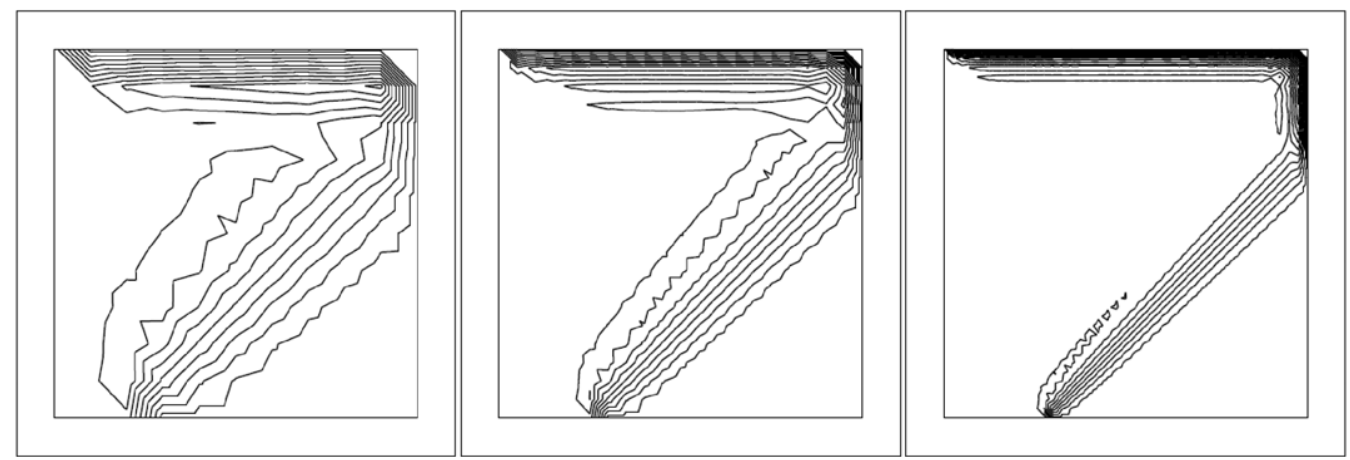

(b)

Fig. 9. Numerical solutions for $\theta=45$ (left to right $N=10,20,40$ ) and the corresponding contour plots. 

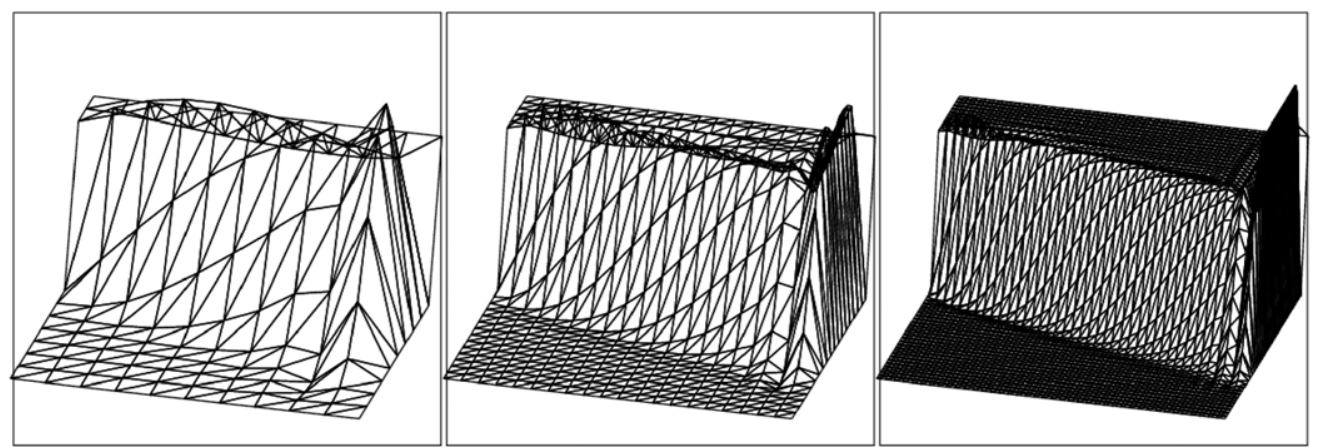

(a)
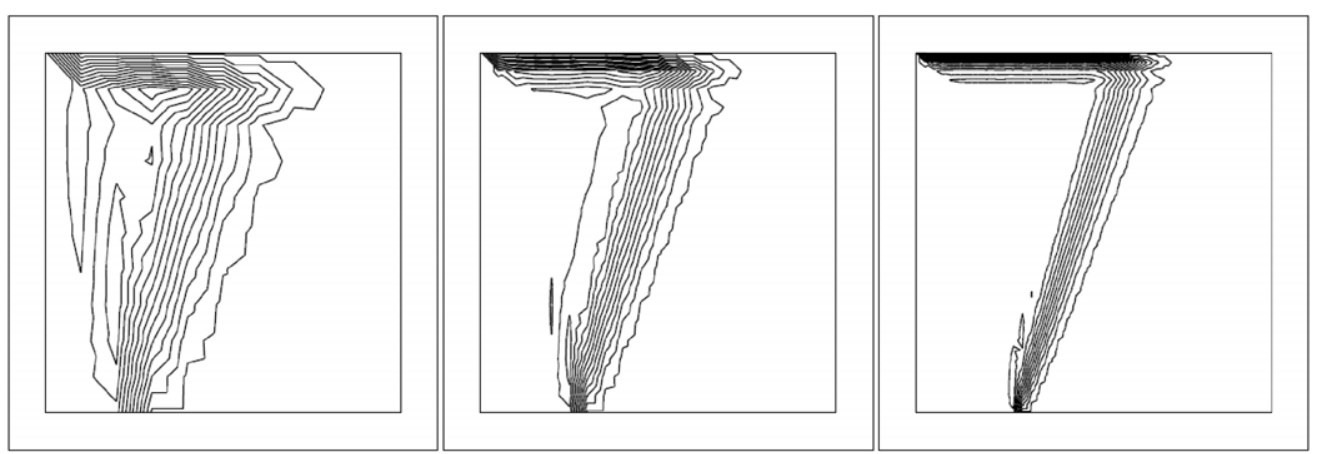

(b)

Fig. 10. Numerical solutions for $\theta=72$ (left to right $N=10,20,40$ ) and the corresponding contour plots.
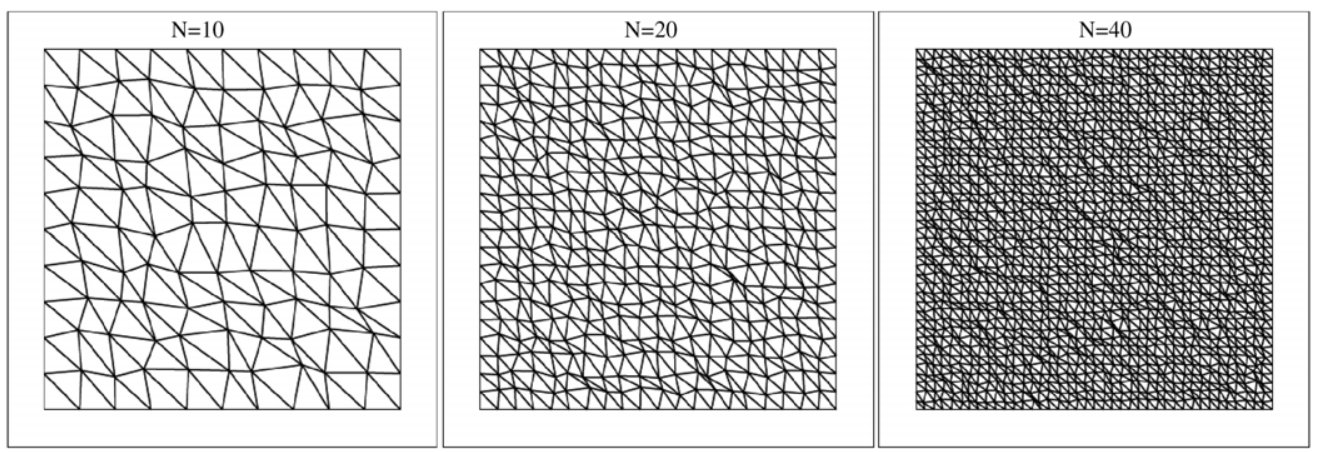

(a)

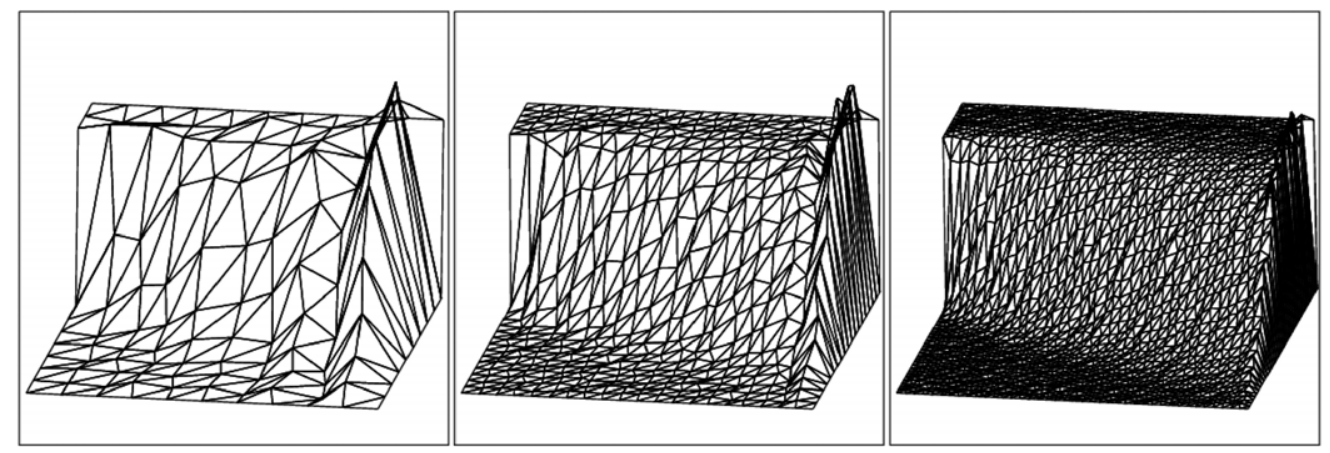

(b)

Fig. 11. (a) Triangular elements used in discretization of the problem domain and (b) numerical solutions for $\epsilon=0.01, \theta=72$ (left to right $N=10,20,40)$. 

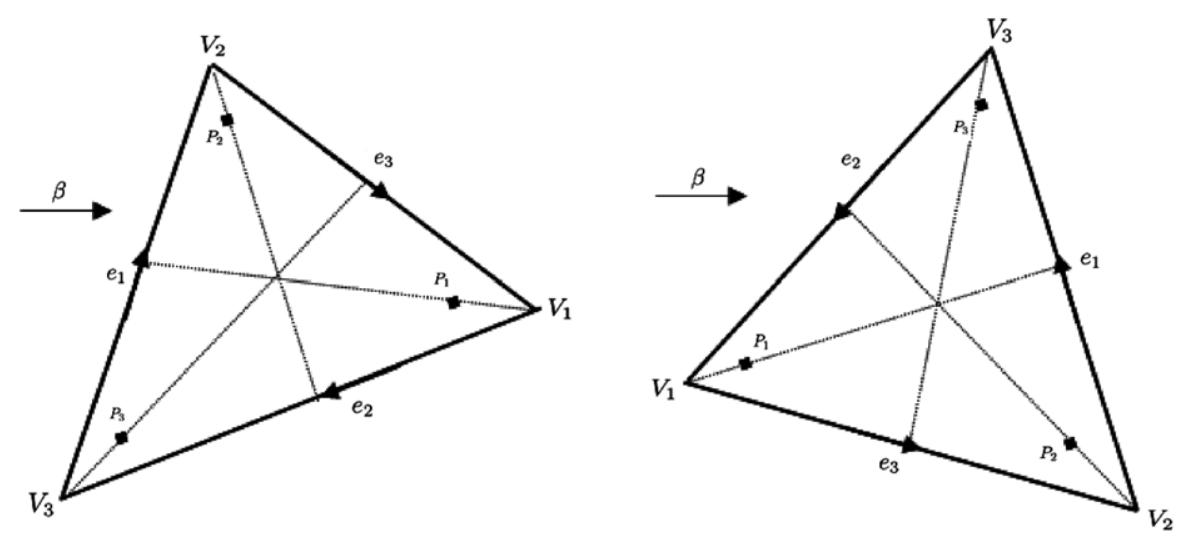

Fig. 12. Configuration of internal nodes for the reaction-dominated regime: one inflow edge (left) and two inflow edges (right).

\section{Reaction-dominated flows}

Now, we analyze the case in which the reaction dominates. This time, we distinguish among the following cases: (see Fig. 12).

\subsection{Two inflow edges}

Let the inflow boundary make up of two edges and let $e_{2}$ and $e_{3}$ be the inflow ones. We assume that the problem is reaction-dominated if

$$
\epsilon \leq \min \left\{\epsilon_{2}^{*}, \epsilon_{3}^{*}\right\} \quad \text { with } \sigma|K|>3 \beta_{\nu_{1}} .
$$

Here, we take the positions of $P_{2}$ and $P_{3}$ as suggested in Section 4 for the convection-dominated regime. Therefore, it only remains us to find a proper location for $P_{1}$. The position of $P_{1}$ along the median from $V_{1}$ is determined by condition (11). Before applying (11), we again split $K$ into three subregions by $P_{1}$ so that we have $\left|K_{11}\right|=t_{1}|K|$ and $\left|K_{12}\right|=\left|K_{13}\right|=\left(1-t_{1}\right)|K| / 2$. Similarly, we have

$$
\alpha_{1}=-\frac{t_{1}\left(1-t_{1}\right)|K|\left(S_{1}+t_{1} \sigma|K|\right)}{f_{123}\left(t_{1}\right)}
$$

where $S_{1}=\sigma|K|-2 \beta_{v_{1}}$. Note that $S_{1}>0$ and $\alpha_{1}<0$ by condition (22). Before finding an optimal position for $P_{1}$, we need the following two intermediate results.

Lemma 5. Let $\alpha_{1}$ be as in (23). Then, we have

$$
1+\alpha_{1}>\frac{1-t_{1}}{2}
$$

Proof. With the use of the explicit form of $\alpha_{1}$ in (23), we have

$$
1+\alpha_{1}-\frac{1-t_{1}}{2}=1-\frac{t_{1}\left(1-t_{1}\right)|K|\left(S_{1}+t_{1} \sigma|K|\right)}{f_{123}\left(t_{1}\right)}-\frac{1-t_{1}}{2}=\frac{4 t\left(1-t_{1}\right) \beta_{\nu_{1}}|K|+3 \epsilon\left(1+t_{1}\right) l_{123}\left(t_{1}\right)}{2 f_{123}\left(t_{1}\right)}
$$

Hence the result immediately follows since $f_{123}\left(t_{1}\right)>0$ whenever $0<t_{1}<1$.

Lemma 6. In reaction-dominated flows, $\int_{K}\left|-\epsilon \Delta B_{1}^{*}\right|$ is an increasing function of $t_{1}$ in the interval $(1 / 2,1)$.

Proof. Following the lines of Lemma 1, it is easily done.

The following lemma suggests an optimal position for $P_{1}$ by using (11). 
Theorem 7. If the inflow boundary make up of two edges, then the point

$$
t_{1}^{* *}=1+\frac{\rho_{1}-\sqrt{\rho_{1}^{2}+\lambda_{1}}}{2 \sigma|K|^{2}}
$$

minimizes the integral $J_{1}$ in reaction-dominated flows.

Proof. According to [31], it is possible to rewrite the integral $J_{1}$ in (11) as follows:

$$
J_{1}=\int_{K}\left|-\epsilon \Delta B_{1}^{*}\right|+\sum_{i=1}^{3} \int_{K_{1 i}}\left|g_{1}\right| .
$$

Without loss of generality, we may assume that $\beta_{\nu_{2}}>\beta_{\nu_{3}}$ and

$$
2 \sigma\left|K_{13}\right|>-4 \beta_{\nu_{3}}+\beta_{\nu_{1}}
$$

in reaction-dominated flows. A direct calculation over $K$ gives,

$$
\begin{aligned}
\int_{K} g_{1} & =-\frac{1}{2} \beta_{\nu_{1}}+\frac{\sigma|K|}{3}\left(\alpha_{1}+1\right) \\
& =-\frac{1}{2} \beta_{\nu_{1}}+\frac{\sigma|K|}{3}\left(-\frac{t_{1}\left(1-t_{1}\right)|K|\left(S_{1}+t_{1} \sigma|K|\right)}{f_{123}\left(t_{1}\right)}+1\right) \\
& =\frac{3 \epsilon l_{123}\left(t_{1}\right)\left(2 \sigma|K|-3 \beta_{\nu_{1}}\right)+2 t_{1}\left(1-t_{1}\right) \sigma|K|^{2}\left(-\beta_{v_{1}}+\left(1-t_{1}\right) \sigma|K|\right)}{6 f_{123}\left(t_{1}\right)}
\end{aligned}
$$

which is positive since $f_{123}\left(t_{1}\right)>0$ and

$$
\begin{aligned}
& 2 \sigma|K|-3 \beta_{v_{1}}>6 \beta_{v_{1}}-3 \beta_{v_{1}}=3 \beta_{v_{1}}>0 \quad \text { (by condition (22)) } \\
& -\beta_{v_{1}}+\left(1-t_{1}\right) \sigma|K|=-\beta_{\nu_{1}}+2 \sigma\left|K_{13}\right|>0 \quad \text { (by assumption (25)). }
\end{aligned}
$$

Now, if we split $K$ into three subregions by $P_{1}$ and calculate the integral of $g_{1}$ over each of these sub-domains, we get

$$
\begin{aligned}
\int_{K_{12}} g_{1} & =-\frac{\alpha_{1}}{2} \beta_{\nu_{2}}-\frac{\left|K_{12}\right|}{2|K|} \beta_{\nu_{1}}+\frac{\sigma\left|K_{12}\right|}{3}\left(\alpha_{1}+\frac{\left|K_{11}\right|}{|K|}+1\right) \\
& >-\frac{\alpha_{1}}{2} \beta_{\nu_{3}}-\frac{\left|K_{12}\right|}{2|K|} \beta_{\nu_{1}}+\frac{\sigma\left|K_{12}\right|}{3}\left(\alpha_{1}+\frac{\left|K_{11}\right|}{|K|}+1\right) \quad\left(\text { since } \beta_{\nu_{2}}>\beta_{\nu_{3}}\right) \\
& =-\frac{\alpha_{1}}{2} \beta_{\nu_{3}}-\frac{\left|K_{13}\right|}{2|K|} \beta_{\nu_{1}}+\frac{\sigma\left|K_{13}\right|}{3}\left(\alpha_{1}+\frac{\left|K_{11}\right|}{|K|}+1\right)=\int_{K_{13}} g_{1}
\end{aligned}
$$

and that

$$
\begin{aligned}
\int_{K_{13}} g_{1} & =-\frac{\alpha_{1}}{2} \beta_{v_{3}}-\frac{\left|K_{13}\right|}{2|K|} \beta_{v_{1}}+\frac{\sigma\left|K_{13}\right|}{3}\left(\alpha_{1}+\frac{\left|K_{11}\right|}{|K|}+1\right) \\
& =-\frac{\alpha_{1}}{2} \beta_{v_{3}}+\frac{\sigma\left|K_{13}\right|}{3}\left(\alpha_{1}+1\right)+\frac{\left|K_{13}\right|}{|K|}\left(\frac{-\beta_{v_{1}}}{2}+\frac{\sigma\left|K_{11}\right|}{3}\right) \\
& >\frac{\beta_{v_{3}}}{2} \frac{1+t_{1}}{2}+\frac{\sigma\left|K_{13}\right|}{3} \frac{1-t_{1}}{2}+\frac{\left|K_{13}\right|}{|K|}\left(\frac{-\beta_{v_{1}}}{2}+\frac{\sigma\left|K_{11}\right|}{3}\right) \quad \text { (by Lemma 5) } \\
& =\frac{\beta_{v_{3}}}{2} \frac{1+t_{1}}{2}+\sigma|K| \frac{\left(1-t_{1}\right)^{2}}{12}+\frac{1-t_{1}}{2}\left(\frac{-\beta_{v_{1}}}{2}+\frac{t_{1}}{3} \sigma|K|\right) \\
& =\frac{\beta_{v_{3}}}{2} \frac{1+t_{1}}{2}-\frac{\beta_{v_{1}}}{2} \frac{1-t_{1}}{2}+\sigma|K| \frac{\left(1-t_{1}\right)\left(1+t_{1}\right)}{12}
\end{aligned}
$$




$$
\begin{aligned}
& >\frac{\beta_{v_{3}}}{2}-\frac{\beta_{v_{1}}}{8}+\sigma|K| \frac{1-t_{1}}{8} \quad\left(\text { since } 1 / 2<t_{1}<1\right) \\
& =\frac{1}{8}\left(4 \beta_{\nu_{3}}-\beta_{v_{1}}+2 \sigma\left|K_{13}\right|\right)
\end{aligned}
$$

which is positive by assumption (25). Thus the second term on the right hand side of (24) attains its minimum if $\int_{K_{11}} g_{1}$ is non-negative, too:

$$
\begin{aligned}
\int_{K_{11}} g_{1} & =-\frac{\alpha_{1}}{2} \beta_{\nu_{1}}-\frac{\left|K_{11}\right|}{2|K|} \beta_{\nu_{1}}+\frac{\sigma\left|K_{11}\right|}{3}\left(\alpha_{1}+\frac{\left|K_{11}\right|}{|K|}\right) \\
& =\left(\alpha_{1}+\frac{\left|K_{11}\right|}{|K|}\right)\left(-\frac{\beta_{\nu_{1}}}{2}+\frac{\sigma\left|K_{11}\right|}{3}\right) \\
& =\left(\alpha_{1}+t_{1}\right)\left(-\frac{\beta_{\nu_{1}}}{2}+\frac{t_{1} \sigma|K|}{3}\right) \\
& =-\frac{t_{1}\left(-3 \beta_{\nu_{1}}+2 \sigma|K| t_{1}\right)\left(t_{1}^{2} \sigma|K|^{2}-t_{1}\left(\rho_{1}+2 \sigma|K|^{2}\right)+|K| S_{1}-3 \epsilon\left|e_{1}\right|^{2}\right)}{6 f_{123}\left(t_{1}\right)} \\
& =-\frac{2 t_{1} \sigma^{2}|K|^{3}\left(t_{1}-\frac{3 \beta_{\nu_{1}}}{2 \sigma|K|}\right)\left(t_{1}-1-\frac{\rho_{1}-\sqrt{\rho_{1}^{2}+\lambda_{1}}}{2 \sigma|K|^{2}}\right)\left(t_{1}-1-\frac{\rho_{1}+\sqrt{\rho_{1}^{2}+\lambda_{1}}}{2 \sigma|K|^{2}}\right)}{6 f_{123}\left(t_{1}\right)} .
\end{aligned}
$$

The only root of the last expression in $(0,1)$ is $t_{1}^{* *}=1+\frac{\rho_{1}-\sqrt{\rho_{1}^{2}+\lambda_{1}}}{2 \sigma|K|^{2}}$ and thus, $\int_{K_{11}} g_{1}$ is positive if $t_{1} \geq t_{1}^{* *}$. This fact together with Lemma 6 determines an optimal value for $t_{1}^{* *}$.

Remark 10. Note that

$$
\lim _{\sigma \rightarrow 0} t_{1}^{* *}=-\infty \quad \text { and } \quad \lim _{\sigma \rightarrow \infty} t_{1}^{* *}=1 .
$$

Thus the points $P_{1}, P_{2}$ and $P_{3}$ should be chosen as

$$
t_{2}=t_{2}^{*}, \quad t_{3}=t_{3}^{*}, \quad t_{1}=\max \left\{\min \left\{1 / 3,1-t_{2}, 1-t_{3}\right\}, t_{1}^{* *}\right\}
$$

where we borrow $t_{2}^{*}$ and $t_{3}^{*}$ from Section 4 .

\subsection{One inflow edge}

In this case, we let $e_{1}$ be the inflow edge and assume that the problem is reaction-dominated under the following conditions

$$
\epsilon \leq \epsilon_{1}^{*} \quad \text { with } \sigma|K|>3 \max \left\{\beta_{\nu_{2}}, \beta_{\nu_{3}}\right\} .
$$

Here, we take the position of $P_{1}$ from the convection-dominated regime suggested in Section 4 and it remains us to find proper locations for $P_{2}$ and $P_{3}$.

\subsubsection{Construction of $P_{2}$ and $P_{3}$}

In the same manner, we determine the locations of $P_{2}$ and $P_{3}$ along the medians from $V_{2}$ and $V_{3}$, respectively. Splitting $K$ into three subregions by $P_{2}$ and $P_{3}$, we have

$$
\begin{aligned}
\left|K_{21}\right| & =\left|K_{23}\right|=\left(1-t_{2}\right)|K| / 2, & & \left|K_{22}\right|=t_{2}|K| . \\
\left|K_{31}\right| & =\left|K_{32}\right|=\left(1-t_{3}\right)|K| / 2, & & \left|K_{33}\right|=t_{3}|K| .
\end{aligned}
$$

Similarly, we get explicit expressions for $\alpha_{2}$ and $\alpha_{3}$ :

$$
\alpha_{2}=-\frac{t_{2}\left(1-t_{2}\right)|K|\left(S_{2}+t_{2} \sigma|K|\right)}{f_{213}\left(t_{2}\right)} \quad \text { and } \quad \alpha_{3}=-\frac{t_{3}\left(1-t_{3}\right)|K|\left(S_{3}+t_{3} \sigma|K|\right)}{f_{312}\left(t_{3}\right)}
$$


where $S_{2}=\sigma|K|-2 \beta_{\nu_{2}}$ and $S_{3}=\sigma|K|-2 \beta_{\nu_{3}}$. Note that $S_{2}>0, \alpha_{2}<0$ and $S_{3}>0, \alpha_{3}<0$ by condition (26). Before finding the optimal positions for $P_{2}$ and $P_{3}$, we need the following two intermediate results.

Lemma 8. Let $\alpha_{2}$ and $\alpha_{3}$ be as in (27). Then, we have

$$
1+\alpha_{2}>\frac{1-t_{2}}{2} \text { and } 1+\alpha_{3}>\frac{1-t_{3}}{2} \text {. }
$$

Proof. The proof is similar to Lemma 5, so we skip it.

Lemma 9. In reaction-dominated flows, $\int_{K}\left|-\epsilon \Delta B_{2}^{*}\right|$ and $\int_{K}\left|-\epsilon \Delta B_{3}^{*}\right|$ are increasing functions of $t_{2}$ and $t_{3}$, respectively, in the interval $(1 / 2,1)$.

Proof. Following the lines of Lemma 6, it is easily done.

Now we are ready to determine optimal positions for $P_{2}$ and $P_{3}$.

Theorem 10. If the inflow boundary make up of one edge, then the points $t_{2}^{* *}=1+\frac{\rho_{2}-\sqrt{\rho_{2}^{2}+\lambda_{2}}}{2 \sigma|K|^{2}}$ and $t_{3}^{* *}=$ $1+\frac{\rho_{3}-\sqrt{\rho_{3}^{2}+\lambda_{3}}}{2 \sigma|K|^{2}}$ minimize the integrals $J_{2}$ and $J_{3}$, respectively, in reaction-dominated flows.

Proof. The proof is similar to the one in Theorem 7. Therefore we point out the major steps. Let us recall that

$$
J_{2}=\int_{K}\left|-\epsilon \Delta B_{2}^{*}\right|+\sum_{i=1}^{3} \int_{K_{2 i}}\left|g_{2}\right| .
$$

Without loss of generality, we may assume that

$$
2 \sigma\left|K_{21}\right|>-4 \beta_{\nu_{1}}+\beta_{\nu_{2}}
$$

in reaction-dominated flows. Then a direct calculation over $K$ gives,

$$
\begin{aligned}
\int_{K} g_{2} & =-\frac{1}{2} \beta_{\nu_{2}}+\frac{\sigma|K|}{3}\left(\alpha_{2}+1\right) \\
& =\frac{3 \epsilon l_{213}\left(t_{2}\right)\left(2 \sigma|K|-3 \beta_{\nu_{2}}\right)+2 t_{2}\left(1-t_{2}\right) \sigma|K|^{2}\left(-\beta_{\nu_{2}}+\left(1-t_{2}\right) \sigma|K|\right)}{6 f_{213}\left(t_{2}\right)}
\end{aligned}
$$

which is positive since $f_{213}\left(t_{2}\right)>0$ and

$$
\begin{aligned}
& 2 \sigma|K|-3 \beta_{\nu_{2}}>6 \beta_{\nu_{2}}-3 \beta_{\nu_{2}}=3 \beta_{\nu_{2}}>0 \quad \text { (by condition (26)) } \\
& -\beta_{\nu_{2}}+\left(1-t_{2}\right) \sigma|K|=-\beta_{\nu_{2}}+2 \sigma\left|K_{21}\right|>0 \quad \text { (by assumption (29)). }
\end{aligned}
$$

Now split $K$ into three subregions by $P_{2}$ as in Fig. 1 and calculate the integral of $g_{2}$ over each of these sub-domains to get

$$
\int_{K_{21}} g_{2}=-\frac{\alpha_{2}}{2} \beta_{\nu_{1}}-\frac{\left|K_{21}\right|}{2|K|} \beta_{\nu_{2}}+\frac{\sigma\left|K_{21}\right|}{3}\left(\alpha_{2}+\frac{\left|K_{22}\right|}{|K|}+1\right)=\frac{1}{8}\left(4 \beta_{\nu_{1}}-\beta_{\nu_{2}}+2 \sigma\left|K_{21}\right|\right)>0
$$

by assumption (29), and that

$$
\begin{aligned}
\int_{K_{23}} g_{2} & =-\frac{\alpha_{2}}{2} \beta_{v_{3}}-\frac{\left|K_{23}\right|}{2|K|} \beta_{\nu_{2}}+\frac{\sigma\left|K_{23}\right|}{3}\left(\alpha_{2}+\frac{\left|K_{22}\right|}{|K|}+1\right) \\
& =-\frac{\alpha_{2}}{2} \beta_{v_{3}}+\frac{\sigma\left|K_{23}\right|}{3}\left(\alpha_{2}+1\right)+\frac{\left|K_{23}\right|}{|K|}\left(-\frac{\beta_{\nu_{2}}}{2}+\frac{\sigma\left|K_{22}\right|}{3}\right) \\
& >-\frac{\alpha_{2}}{2} \beta_{v_{3}}+\frac{\sigma\left|K_{23}\right|}{3} \frac{1-t_{2}}{2}+\frac{\left|K_{23}\right|}{|K|}\left(-\frac{\beta_{\nu_{2}}}{2}+\frac{\sigma\left|K_{22}\right|}{3}\right) \quad \text { (by Lemma 8) }
\end{aligned}
$$



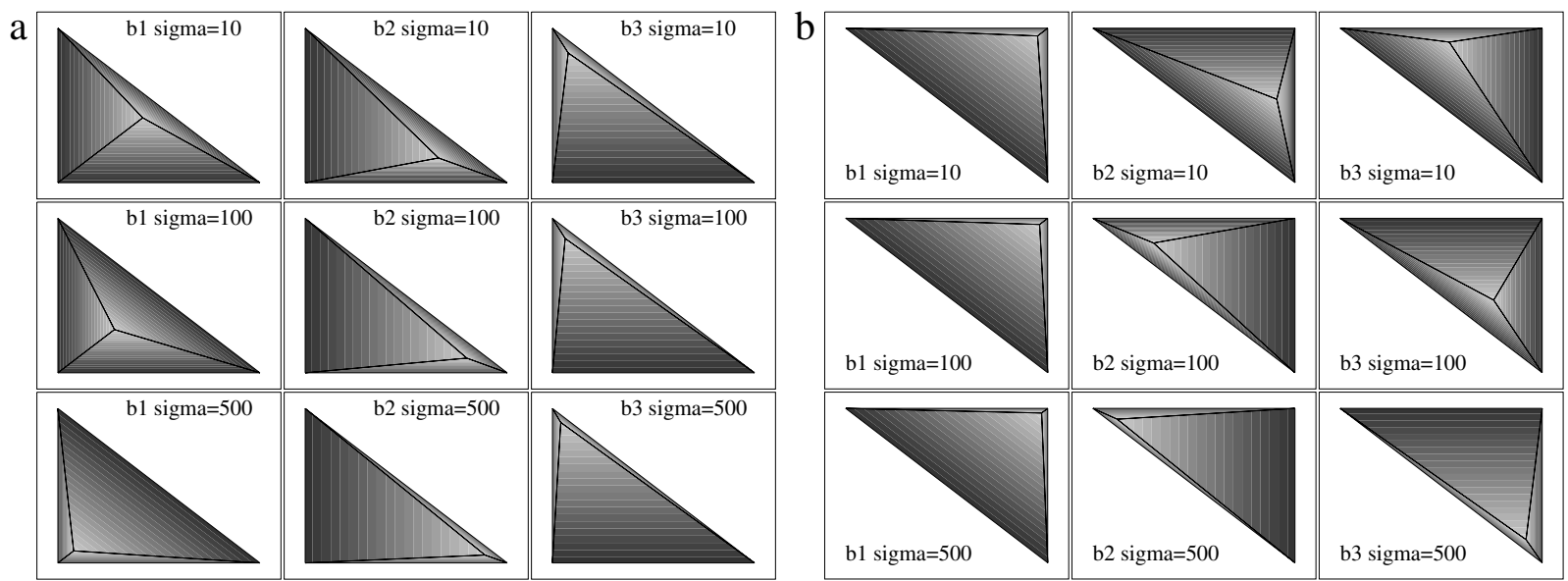

Fig. 13. Bubble functions, $b_{1}, b_{2}, b_{3}$, in a typical element having (a) two inflow edges, (b) one inflow edge, when $\epsilon=10^{-3}, \theta=72^{\circ}, N=10$ and $\sigma=10,100,500$.

which is also positive by condition (26). Thus the second term on the right hand side of (28) attains its minimum if $\int_{K_{22}} g_{2}$ is non-negative, too:

$$
\begin{aligned}
\int_{K_{22}} g_{2} & =-\frac{\alpha_{2}}{2} \beta_{\nu_{2}}-\frac{\left|K_{22}\right|}{2|K|} \beta_{\nu_{2}}+\frac{\sigma\left|K_{22}\right|}{3}\left(\alpha_{2}+\frac{\left|K_{22}\right|}{|K|}\right) \\
& =\left(\alpha_{2}+t_{2}\right)\left(-\frac{\beta_{\nu_{2}}}{2}+\frac{t_{2} \sigma|K|}{3}\right) \\
& =-\frac{2 t_{2} \sigma^{2}|K|^{3}\left(t_{2}-\frac{3 \beta_{\nu_{2}}}{2 \sigma|K|}\right)\left(t_{2}-1-\frac{\rho_{2}-\sqrt{\rho_{2}^{2}+\lambda_{2}}}{2 \sigma|K|^{2}}\right)\left(t_{2}-1-\frac{\rho_{2}+\sqrt{\rho_{2}^{2}+\lambda_{2}}}{2 \sigma|K|^{2}}\right)}{6 f_{213}\left(t_{2}\right)} .
\end{aligned}
$$

The only root of the last expression in $(0,1)$ is $t_{2}^{* *}=1+\frac{\rho_{2}-\sqrt{\rho_{2}^{2}+\lambda_{2}}}{2 \sigma|K|^{2}}$ and thus, $\int_{K_{22}} g_{2}$ is positive if $t_{2} \geq t_{2}^{* *}$. This fact together with Lemma 9 determines an optimal value for $t_{2}^{* *}$.

The proof for $t_{3}^{* *}$ is similarly accomplished by just changing the roles of $e_{2}$ and $e_{3}$ and replacing subindex 2 by subindex 3 accordingly.

Remark 11. Note that

$$
\begin{aligned}
& \lim _{\sigma \rightarrow 0} t_{2}^{* *}=-\infty \quad \text { and } \quad \lim _{\sigma \rightarrow \infty} t_{2}^{* *}=1 \\
& \lim _{\sigma \rightarrow 0} t_{3}^{* *}=-\infty \quad \text { and } \quad \lim _{\sigma \rightarrow \infty} t_{3}^{* *}=1 .
\end{aligned}
$$

Thus the points $P_{1}, P_{2}$ and $P_{3}$ should be taken as

$$
t_{1}=t_{1}^{*}, \quad t_{2}=\max \left\{1-t_{1}, t_{2}^{* *}\right\}, \quad t_{3}=\max \left\{1-t_{1}, t_{3}^{* *}\right\}
$$

where we borrow $t_{1}^{*}$ from Section 4 .

Remark 12. In Fig. 13, we demonstrate the behavior of approximate bubble functions in two types of element configuration with two inflow edges (left) and with one inflow edge (right) for various values of reaction $(\sigma=$ $10,100,500$ ) when $\epsilon=10^{-3}$ and $\theta=72^{\circ}$. The first column of the figure presents the bubble function $b_{1}$, for increasing values of reaction $(\sigma=10,100,500)$. The corresponding numerical results for $b_{2}$ and $b_{3}$ are shown in column 2 and 3, respectively. Note the self-adjustment of the subgrid nodes as the problem evolves from the 


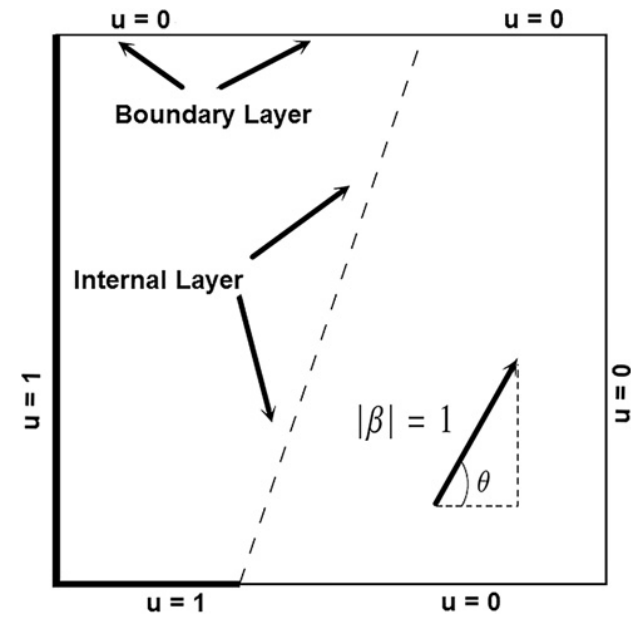

Fig. 14. Configuration of Experiment 1.

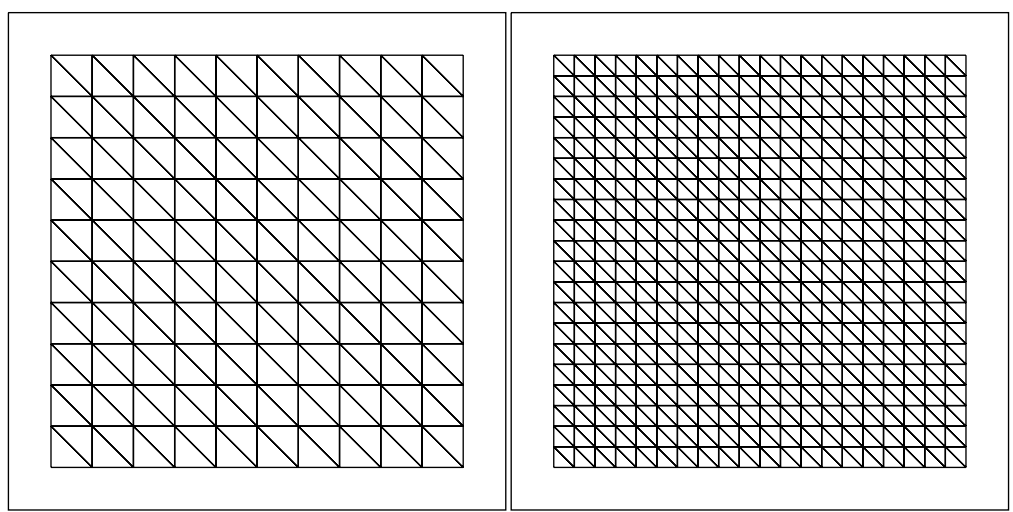

Fig. 15. Triangular elements used in discretization of the problem domain: $N=10$ (left) and $N=20$ (right).

convection-dominated regime into the reaction-dominated one. The locations are readjusted so that the pseudo bubbles contribute to the stability of the numerical method at their most.

\subsection{Numerical experiments}

In this section, we report some numerical experiments to illustrate the performance of the present algorithm in the interesting case of small diffusion which corresponds to the convection-dominated or reaction-dominated regimes depending on the ratio between the related problem parameters.

\subsubsection{Experiment 1}

We start the numerical experiments with a test problem that is taken from [35]. Boundary conditions are displayed in Fig. 14. We take a pair of uniform triangular meshes which are made up of $N=10,20$ elements, respectively, in $x$ and $y$ directions (Fig. 15). In Figures 16-17, we take $\epsilon=10^{-4}$ and $\theta=72^{\circ}$ and plot the solutions obtained with the present method for various values of reaction $(\sigma=f=0.001,1,10,20,50,1000)$. For the smaller values of reaction parameter, the problem falls into the convection-dominated regime. The problem turns into the reaction-dominated one as the reaction parameter increases. So this experiment is designed to test transition between the problem regimes. The method is apparently able to capture the dominant characteristics of the exact solution continuously. 

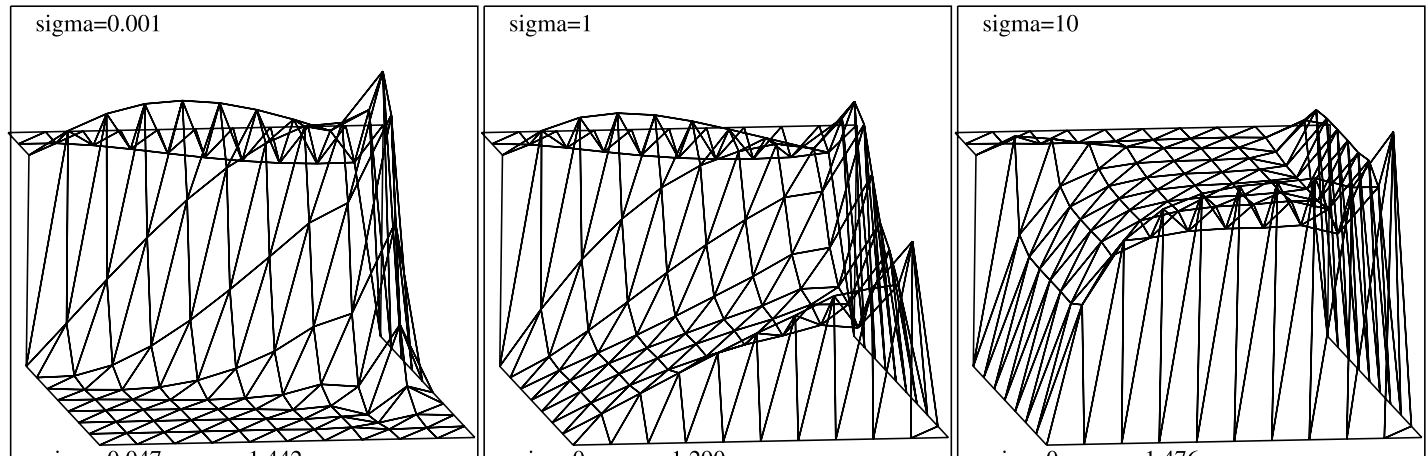

$\min =-0.047, \max =1.442$

$\min =0, \max =1.290$

$\min =0, \max =1.476$
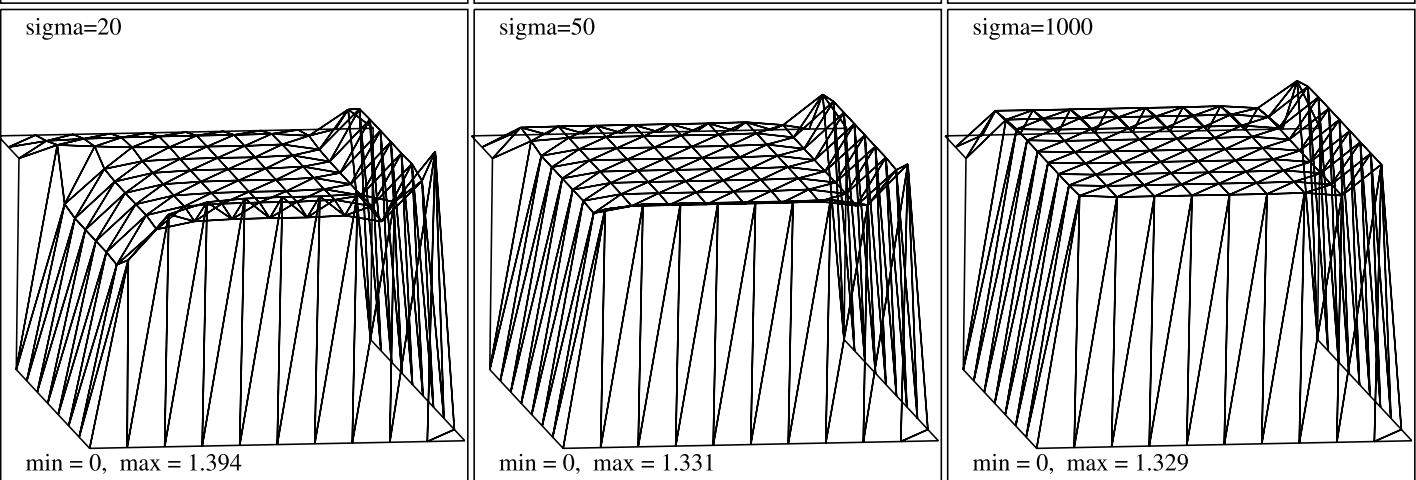

Fig. 16. Numerical solution for $\theta=72^{\circ}, \epsilon=10^{-4}$ and $N=10$.
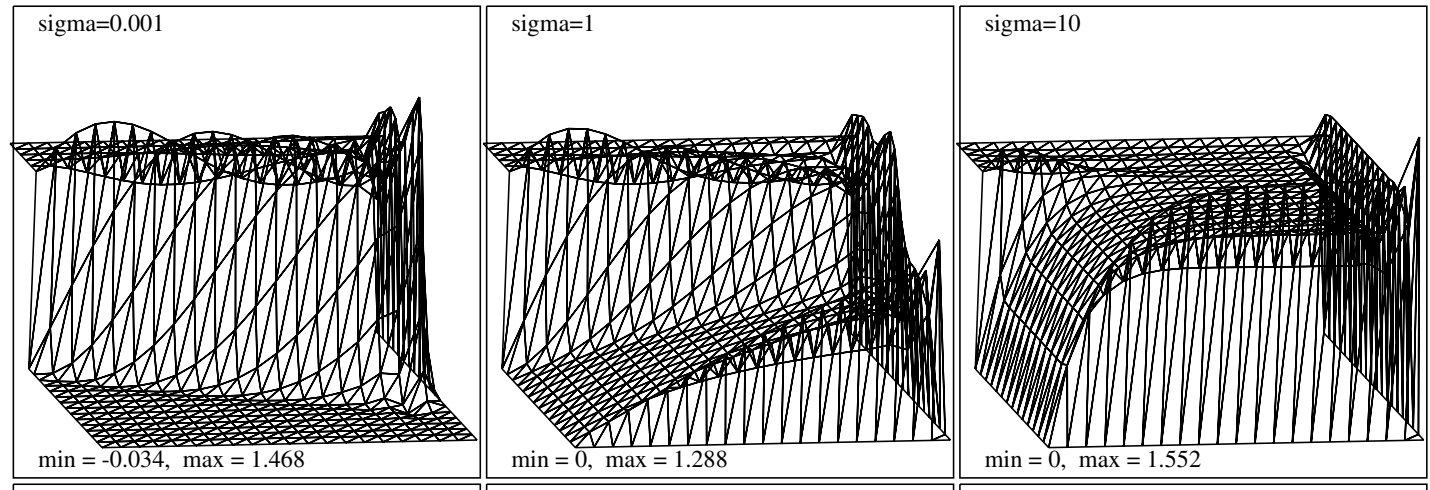

$\min =-0.034, \max =1.468$

$x=1.288$

$\min =0, \max =1.552$
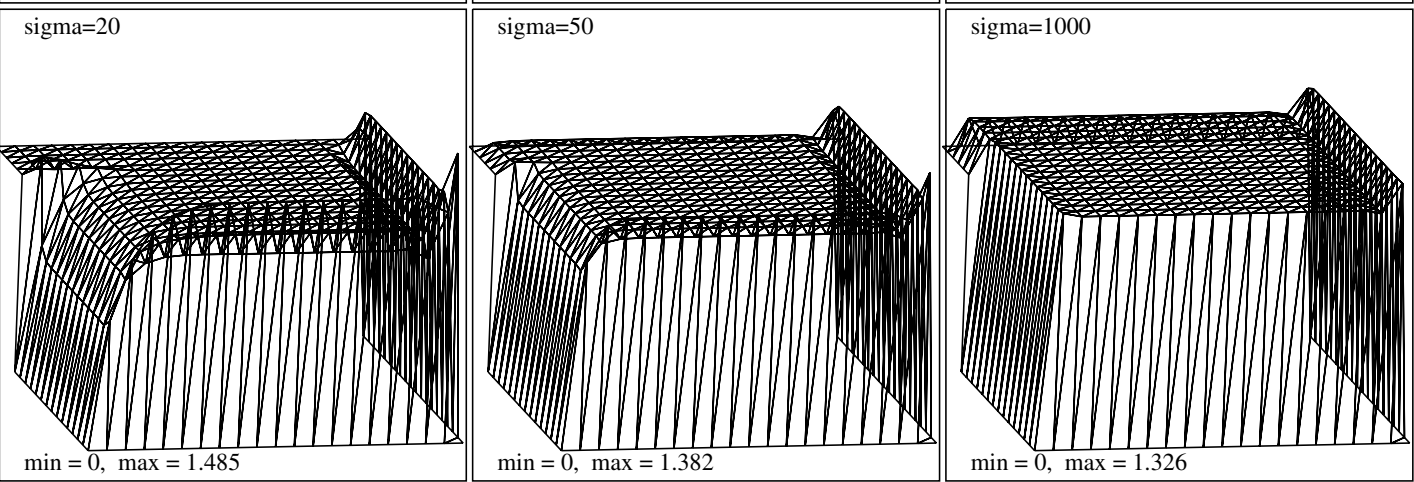

Fig. 17. Numerical solution for $\theta=72^{\circ}, \epsilon=10^{-4}$ and $N=20$. 


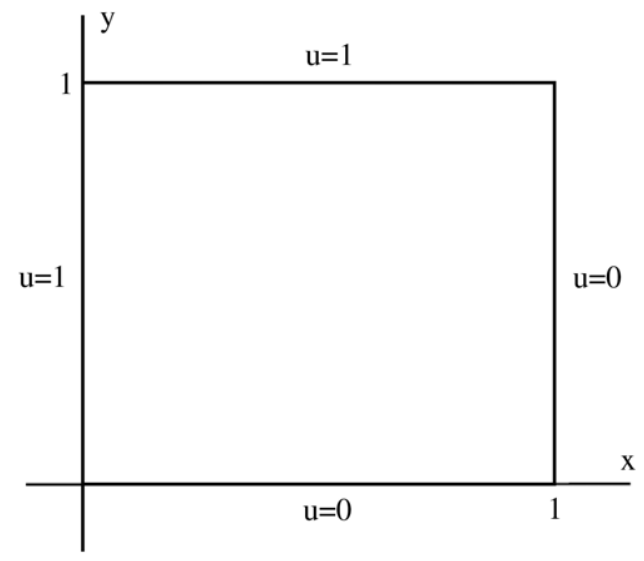

Fig. 18. Configuration of Experiment 2.

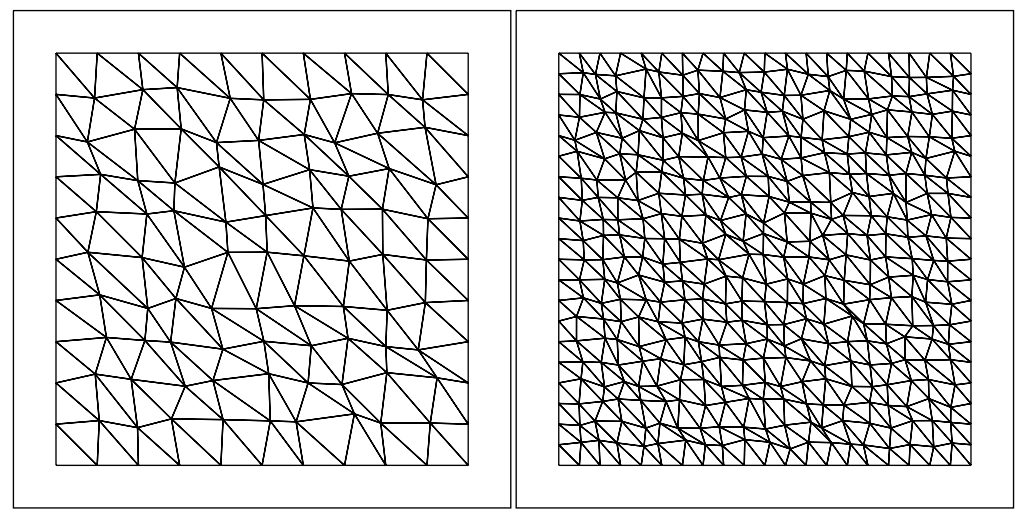

Fig. 19. Triangular elements used in discretization: $N=10$ (left) and $N=20$ (right).

\subsubsection{Experiment 2}

Next, we consider a test problem which is taken from [11]. We display the boundary conditions in Fig. 18 and test it on a pair of uniform and non-uniform triangular meshes, made up of $N=10$ and 20 elements, respectively, in $x$ and $y$ directions (see Fig. 19). In Figs. 20 and 21, we plot the solutions obtained with the present method when $\epsilon=10^{-4}$, $\beta=(0.15,0.1)$ and $f=0$ for various values of reaction $\left(\sigma=10,10^{2}, 10^{3}\right)$. The numerical solutions show that the method is robust as the results are consistent with the physical configuration of the problem on both uniform and non-uniform meshes.

Several numerical examples exhibiting boundary/internal layers are given to illustrate the efficiency and performance of the proposed finite element method. Although the present method could not eliminate the oscillations at all, it captures the layers well like the well-known stabilized methods in the literature, at a lower price compared to the RFB approach. In all cases, it is clear that the proposed method is able to produce the dominant characteristics of the exact solution for the whole range of problem parameters at all levels of the mesh employed. We also note that, the transition from one regime to another is accurately captured by the algorithm.

\section{Conclusion}

A stabilized finite element method for convection-diffusion-reaction problems has been introduced and tested. In particular, we wanted to recover the stabilizing effect of the RFBs at a lower price by approximating them on a specially chosen subgrid and using these approximations in place of RFB functions. The idea behind the choice of the internal nodes is based on minimizing the residual of a set of local differential equations defining the RFBs. Based on 


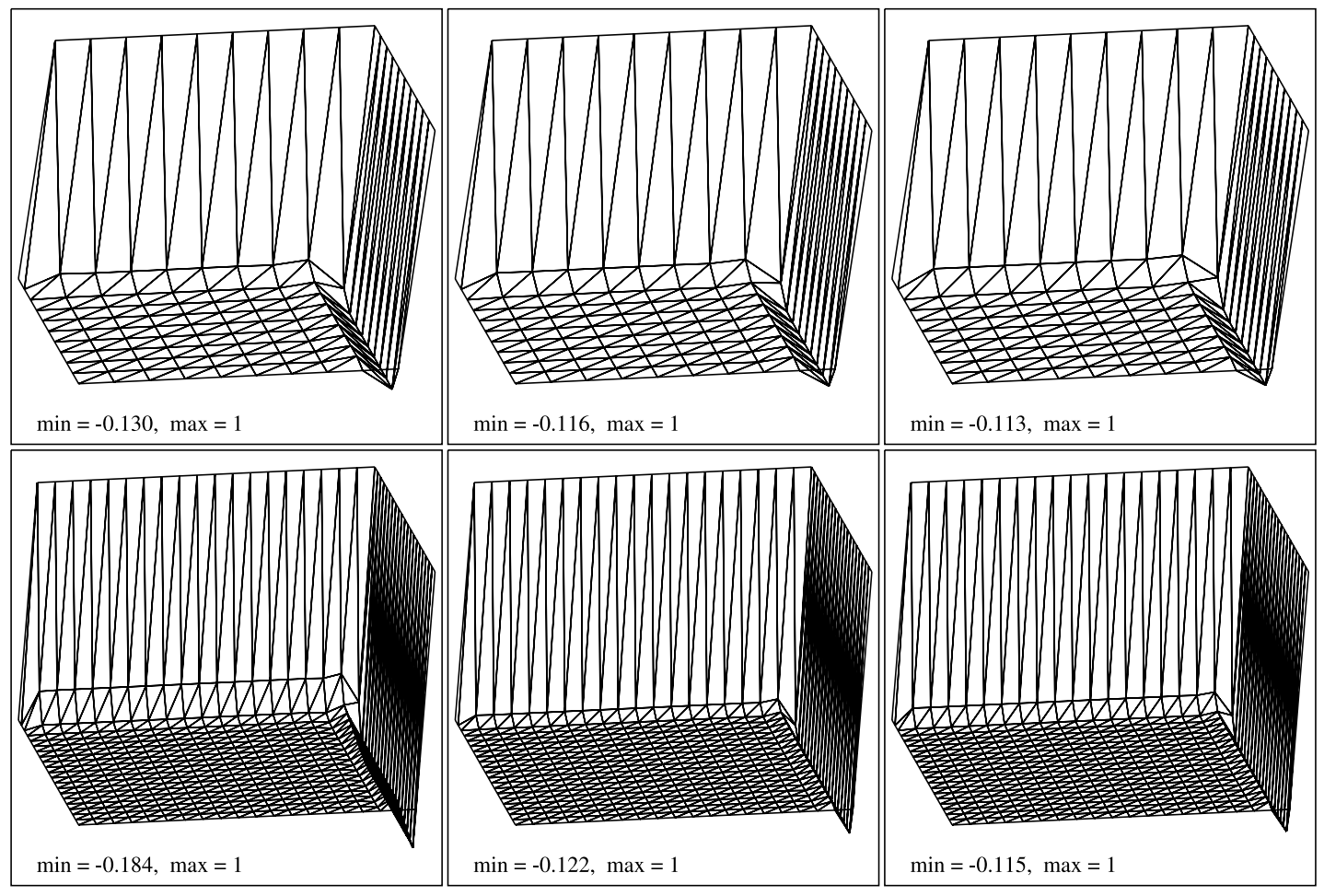

Fig. 20. Numerical solution for $\epsilon=10^{-4}$ (Top to bottom: $N=10,20$ Left to right: $\sigma=10,10^{2}, 10^{3}$ ).

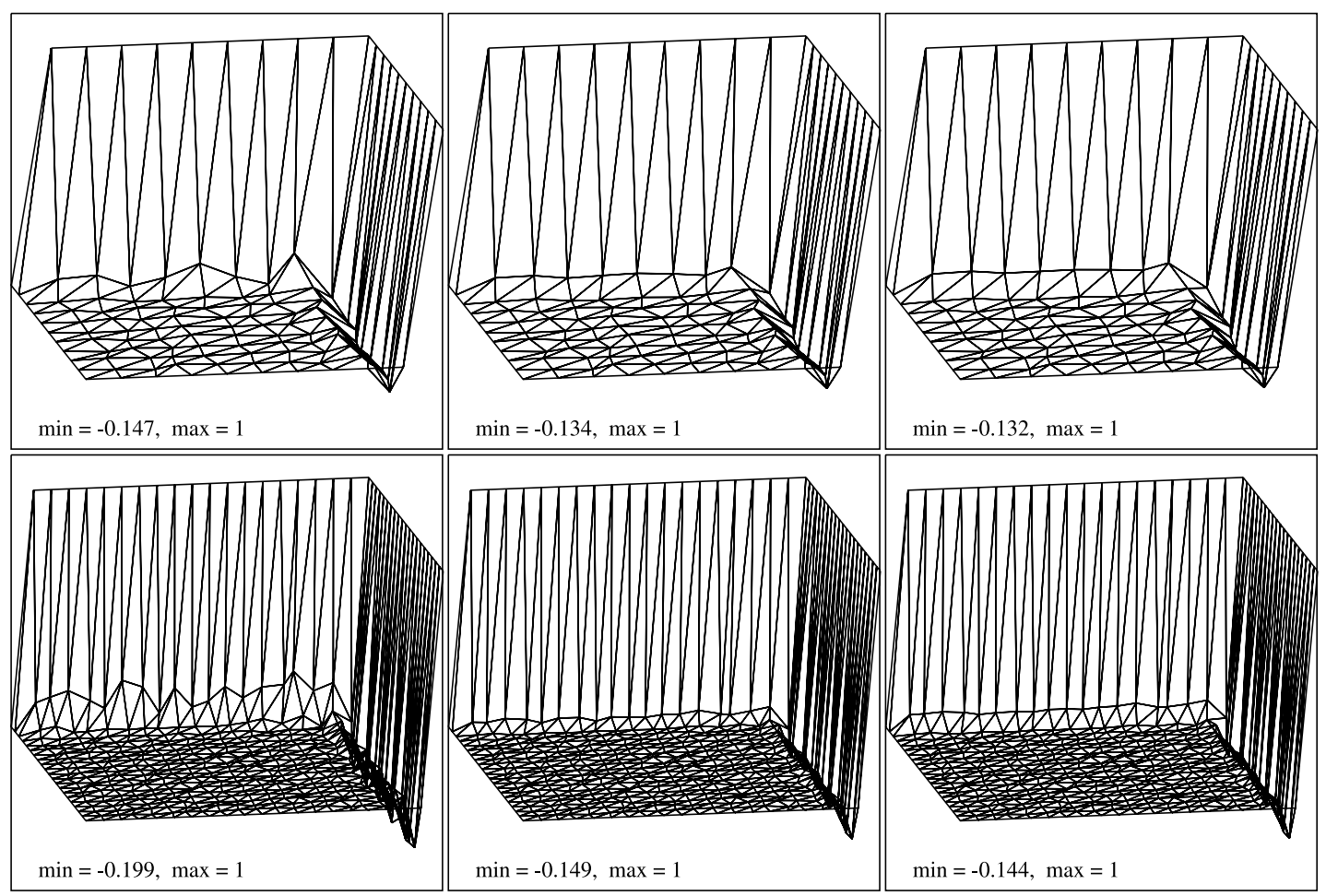

Fig. 21. Top to bottom: $N=10$, 20. Left to right: $\sigma=10,10^{2}, 10^{3}$. 
the numerical experiences on several benchmark problems, we can conclude that the pseudo RFBs retain the stability features of RFBs.

\section{Acknowledgment}

This research was partially done while A. Sendur was a Ph.D. student at the Department of Mathematics, Izmir Institute of Technology.

\section{References}

[1] A.N. Brooks, T.J.R. Hughes, Streamline upwind/Petrov-Galerkin formulations for convection-dominated flows with particular emphasis on the incompressible Navier-Stokes equations, Comput. Methods Appl. Mech. Engrg. 32 (1982) 199-259.

[2] L.P. Franca, S.L. Frey, T.J.R. Hughes, Stabilized finite element methods: I. Application to the advective-diffusive model, Comput. Methods Appl. Mech. Engrg. 95 (1992) 253-276.

[3] T.J.R Hughes, M. Mallet, A. Miznkaml, A new finite element formulation for computational fluid dynamics: II. Beyond SUPG, Comput. Methods Appl. Mech. Engrg. 54 (1986) 341-355.

[4] T.J.R. Hughes, L.P. Franca, G. Hulbert, A new finite element formulation for computational fluid dynamics: VIII. The Galerkin/least-squares method for advective-diffusive equations, Comput. Methods Appl. Mech. Engrg. 73 (1989) 173-189.

[5] L.P. Franca, G. Hauke, A. Masud, Revisiting stabilized finite element methods for the advective-diffusive equation, Comput. Methods Appl. Mech. Engrg. 195 (2006) 1560-1572.

[6] I. Harari, T.J.R. Hughes, Stabilized finite element methods for steady advection-diffusion with production, Comput. Methods Appl. Mech Engrg. 115 (1994) 165-191.

[7] P. Nadukandi, E. Onate, J. Garcia, A high resolution Petrov-Galerkin method for the 1D convection-diffusion-reaction problem, Comput. Methods Appl. Mech. Engrg. 199 (2010) 525-546.

[8] E. Onate, Derivation of stabilized equations for numerical solution of advective-diffusive transport and fluid flow problems, Comput. Methods Appl. Mech. Engrg. 151 (1998) 233-265.

[9] E. Onate, J. Miguel, G. Hauke, Stabilized formulation for advection-diffusion-absorption equation using finite calculus and linear finite elements, Comput. Methods Appl. Mech. Engrg. 195 (2006) 3926-3946.

[10] L.P. Franca, C. Farhat, Bubble functions prompt unusual stabilized finite element methods, Comput. Methods Appl. Mech. Engrg. 123 (1995) 299-308.

[11] L.P. Franca, F. Valentin, On an improved unusual stabilized finite element method for the advective-reactive-diffusive equation, Comput Methods Appl. Mech. Engrg. 190 (2000) 1785-1800.

[12] R. Codina, Comparison of some finite element methods for solving the diffusion-convection-reaction equation, Comput. Methods Appl Mech. Engrg. 156 (1998) 185-210.

[13] T.J.R Hughes, Multiscale phenomena: Green's functions, the Dirichlet-to-Neumann formulation, subgrid scale models, bubbles and the origin of stabilized methods, Comput. Methods Appl. Mech. Engrg. 127 (1995) 387-401.

[14] T.J.R Hughes, G.R. Feijoo, L. Mazzei, J.B. Quincy, The variational multiscale method—a paradigm for computational mechanics, Comput Methods Appl. Mech. Engrg. 166 (1-2) (1998) 3-24.

[15] G. Hauke, A simple subgrid scale stabilized method for the advection-diffusion-reaction equation, Comput. Methods Appl. Mech. Engrg. 191 (2002) 2925-2947.

[16] G. Hauke, A. Garcia-Olivares, Variational subgrid scale formulations for the advection-diffusion-reaction equation, Comput. Methods Appl Mech. Engrg. 190 (2001) 6847-6865.

[17] A. Masud, R. Khurram, A multiscale/stabilized finite element method for the advection-diffusion equation, Comput. Methods Appl. Mech Engrg. 192 (2004) 1997-2018

[18] A. Masud, R. Khurram, A multiscale finite element method for the incompressible Navier-Stokes equations, Comput. Methods Appl. Mech Engrg. 195 (2006) 1750-1777.

[19] F. Brezzi, A. Russo, Choosing bubbles for advection-diffusion problems, Math. Models Methods Appl. Sci. 4 (1994) $571-587$.

[20] F. Brezzi, L.P. Franca, A. Russo, Further considerations on residual-free bubbles for advective-diffusive equations, Comput. Methods Appl. Mech. Engrg. 166 (1998) 25-33.

[21] L.P. Franca, A. Nesliturk, M. Stynes, On the stability of residual-free bubbles for convection-diffusion problems and their approximation by a two-level finite element method, Comput. Methods Appl. Mech. Engrg. 166 (1998) 35-49.

[22] L.P. Franca, L. Tobiska, Stability of the residual free bubble method for bilinear finite elements on rectangular grids, IMA J. Numer. Anal. 22 (2002) 73-87.

[23] L.P. Franca, J.V.A. Ramalho, F. Valentin, Multiscale and residual-free bubble functions for reaction-advection-diffusion problems, Int. J. Multiscale Eng. 3 (2005) 297-312.

[24] A. Russo, Streamline-upwind Petrov/Galerkin method (SUPG) vs residual-free bubbles (RFB), Comput. Methods Appl. Mech. Engrg. 195 (2006) 1608-1626.

[25] C. Baiocchi, F. Brezzi, L.P. Franca, Virtual bubbles and the GaLS, Comput. Methods Appl. Mech. Engrg. 105 (1993) $125-141$.

[26] F. Brezzi, M.O. Bristeau, L.P. Franca, M. Mallet, G. Roge, A relationship between stabilized finite element methods and the Galerkin method with bubble functions, Comput. Methods Appl. Mech. Engrg. 96 (1992) 117-129.

[27] F. Brezzi, L.P. Franca, T.J.R. Hughes, A. Russo, $b=\int g$, Comput. Methods Appl. Mech. Engrg. 145 (1997) 329-339. 
[28] F. Brezzi, G. Hauke, D. Marini, G. Sangalli, Link-cutting bubbles for the stabilization of convection-diffusion-reaction problems, Math. Models Methods Appl. Sci. 13 (2003) 445-461.

[29] F. Brezzi, D. Marini, A. Russo, On the choice of a stabilizing sub-grid for convection-diffusion problems, Comput. Methods Appl. Mech. Engrg. 194 (2005) 127-148.

[30] A.I. Nesliturk, On the choice of stabilizing subgrid for convection-diffusion problem on rectangular grids, Comput. Math. Appl. 59 (2010) 3687-3699.

[31] F. Brezzi, D. Marini, A. Russo, Applications of pseudo residual-free bubbles to the stabilization of convection-diffusion problems, Comput. Methods Appl. Mech. Engrg. 166 (1998) 51-63.

[32] A. Nesliturk, A stabilizing sub-grid for convection-diffusion problem, Math. Mod. Meth. Appl. Sci. 16 (2006) $211-231$.

[33] A. Sendur, A.I. Nesliturk, Applications of the pseudo residual-free bubbles to the stabilization of convection-diffusion-reaction problems, Calcolo 49 (2012) 1-19.

[34] L.P. Franca, F. Hwang, Refining the submesh strategy in the two-level finite element method: Application to the advection-diffusion equation, Inter. J. Numer. Methods Fluids 39 (2002) 161-187.

[35] M.I. Asensio, A. Russo, G. Sangalli, The residual-free bubble numerical method with quadratic elements, Math. Models Methods Appl. Sci. 14 (2004) 641-661. 